\title{
Ruthenium-catalyzed Decarboxylative Insertion of Electrophiles.
}

Chao Wang and Jon A. Tunge

\section{Supporting Information: Table of Contents.}

$\begin{array}{ll}\text { General experimental } & \text { S-1 } \\ \text { General procedure for tandem Michael/Allylation } & \text { S-1 } \\ \text { Spectral characterization of } \mathbf{2 a} & \text { S-2 } \\ \text { Spectral characterization of } \mathbf{2 b} & \text { S-3 } \\ \text { Spectral characterization of } \mathbf{2 d} & \text { S-3 } \\ \text { Spectral characterization of } \mathbf{2 e} & \text { S-4 } \\ \text { Spectral characterization of } \mathbf{2 f} & \text { S-5 } \\ \text { Spectral characterization of } \mathbf{2 g} & \text { S-6 } \\ \text { Spectral characterization of } \mathbf{2 h} & \text { S-7 } \\ \text { Spectral characterization of } \mathbf{2 i} & \text { S-7 } \\ \text { Spectral characterization of } \mathbf{3 j} & \text { S-8 } \\ \text { Spectral characterization of } \mathbf{3 k} & \text { S-9 } \\ \text { Spectral characterization of } \mathbf{3 1} & \text { S-11 } \\ \text { Spectral characterization of } \mathbf{4} \mathbf{f} & \text { S-12 } \\ \text { Spectral characterization of } \mathbf{4 i} & \text { S-12 } \\ \text { Spectral characterization of } \mathbf{4 m} & \text { S-13 } \\ \text { Spectral characterization of } \mathbf{5} & \text { S-14 }\end{array}$

\section{Experimental}

Materials. Methylene chloride and tetrahydrofuran were dried over activated alumina. ${ }^{1}$ All other materials were used as received. $\beta$-ketoesters were prepared by DMAP-catalyzed addition of diketene to the corresponding allylic alcohols. ${ }^{2} \quad 4$-substituted $\beta$-ketoesters were prepared by the condensation of the corresponding acid chloride with Meldrum's acid ${ }^{3}$ followed by addition of the appropriate allylic alcohol. ${ }^{4}{ }^{1} \mathrm{H}$ NMR spectra were referenced to residual protio solvent signals. Structural assignments are based on ${ }^{1} \mathrm{H},{ }^{13} \mathrm{C}$, DEPT-135, COSY, and HMQC spectroscopies.

General procedure for catalytic tandem Michael/Allylation(with Ru): In a Schlenk tube under argon, $\left[\mathrm{Cp}^{*} \mathrm{RuCl}\right] 4$ (2.5 mol \%), bipyridine (10 mol \%), allyl- $\beta$-ketoester $(1 \mathrm{mmol})$ and malonitrile $(1 \mathrm{mmol})$ were dissolved in $5 \mathrm{~mL}$ of

\footnotetext{
${ }^{1}$ Pnagborn, A. B.; Giardello, M. A.; Grubbs, R. H; Rosen, R. K.; Timmers, F. J. Organometallics 1996, 15, 1518-20.

${ }^{2}$ Collado, I.; Pedregal, C.; Mazon, A.; Espinosa, J. F.; Blanco-Urgoiti, J.; Schoepp, D. D.; Wright, R. A.; Johnson, B. G.; Kingston, A. E. J. Med.Chem. 2002, 45, 3619-3629.

${ }^{3}$ Svenstrup, N.; Simonsen, K.; Thorup, N.; Brodersen, J.; Dehaen, W.; Becher, J. J. Org. Chem. 1999, 64, 2814-2820.

${ }^{4}$ Yuste, F.; Brena, F.; Barrios, H.; Sanchez-Obregon, R.; Ortiz, B.; Walls, F. Synth. Commun. 1988, $18,735-739$.
} 
methylene chloride. The resulting deep purple solution was stirred at ambient temperature under Ar for the specified times (Table 1 of text). Reaction completion was determined by TLC. Following solvent evaporation the crude product was purified via flash chromatography $\left(\mathrm{SiO}_{2}, 3: 1\right.$ Hexane: $\left.\mathrm{Et}_{2} \mathrm{O}\right)$.

General procedure for catalytic tandem Michael/Allylation(with Pd): In a Schlenk tube under argon, $\left[\mathrm{PdPPh}_{3}\right]_{4}(10 \mathrm{~mol} \%)$, allyl- $\beta$-ketoester $(1 \mathrm{mmol})$, and malonitrile (1 mmol) were dissolved in $5 \mathrm{~mL}$ of methylene chloride. The resulting yellow solution was stirred at ambient temperature under Ar for the specified times (Table 1 of text). Reaction completion was determined by TLC. Following solvent evaporation the crude product was purified via flash chromatography $\left(\mathrm{SiO}_{2}, 3: 1\right.$ Hexane: $\left.\mathrm{Et}_{2} \mathrm{O}\right)$.

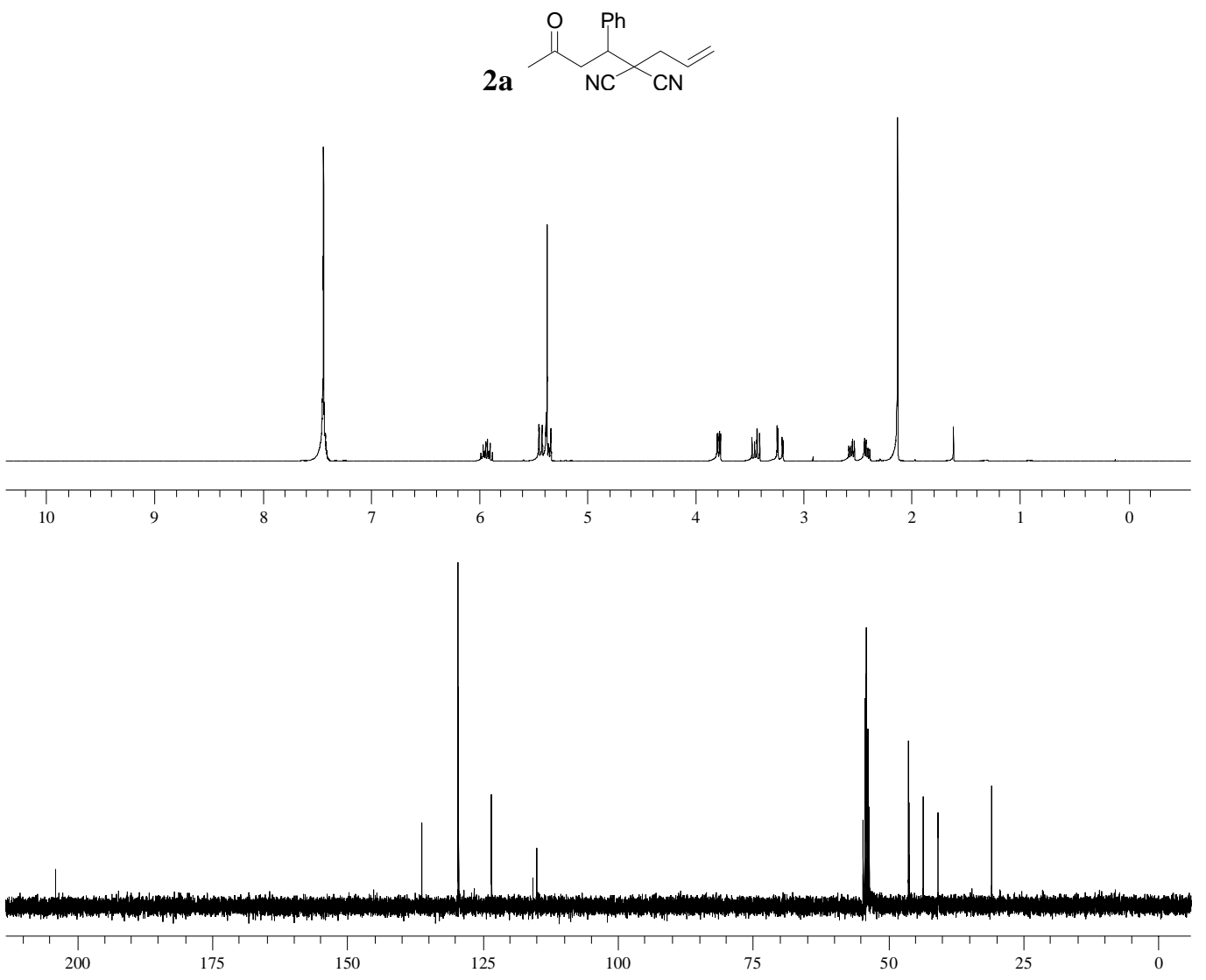

2a: ${ }^{1}$ Oil: ${ }^{1} \mathrm{H}$ NMR (400 MHz, $\left.\mathrm{CD}_{2} \mathrm{Cl}_{2}\right) \delta 7.44(\mathrm{~m}, 5 \mathrm{H}$ : arom $\mathrm{H}), 5.98-5.88(\mathrm{~m}, 1 \mathrm{H}, J=10.1,6.8,7.3$, $7.1 \mathrm{~Hz}:=\mathrm{CH}), 5.43\left(\mathrm{~d}, 1 \mathrm{H}, J=10.1 \mathrm{~Hz}: \mathrm{CH}=\mathrm{CH}(H)_{\mathrm{Z}}\right), 5.23\left(\mathrm{~d}, 1 \mathrm{H}, J=6.8 \mathrm{~Hz}: \mathrm{CH}=\mathrm{CH}(H)_{\mathrm{E}}\right), 3.78$ (dd, $\left.1 \mathrm{H}, J=10.4,3.5 \mathrm{~Hz}: \mathrm{C}(\mathrm{O}) \mathrm{CH}_{2} \mathrm{CHPh}\right), 3.44\left(\mathrm{dd}, J=10.4,17.4 \mathrm{~Hz}, 1 \mathrm{H}: \mathrm{C}(\mathrm{O}) \mathrm{C} \mathbf{H}_{2}\right), 3.22(\mathrm{dd}, J$ $\left.=3.5,17.4 \mathrm{~Hz}, 1 \mathrm{H}: \mathrm{C}(\mathrm{O}) \mathrm{CH}_{2}\right), 2.56\left(\mathrm{dd}, J=7.3,14.1 \mathrm{~Hz}, 1 \mathrm{H}: \mathrm{CH}_{2} \mathrm{CH}=\right), 2.42(\mathrm{dd}, J=7.1,14.1 \mathrm{~Hz}$, 1H: $\left.\mathrm{CH}_{2} \mathrm{CH}=\right), 2.13\left(\mathrm{~s}, 3 \mathrm{H}: \mathrm{C}(\mathrm{O}) \mathrm{CH}_{3}\right) ;{ }^{13} \mathrm{C}$ NMR $\left(100 \mathrm{MHz}, \mathrm{CD}_{2} \mathrm{Cl}_{2}\right) \delta 203.95(\mathbf{C}=\mathrm{O}), 136.15$ (Quat.), $129.54(=\mathbf{C H}), 129.54$ (Arom. $\mathbf{C H}), 123.36\left(=\mathrm{CH}_{2}\right), 115.66(\mathbf{C N}), 114.91(\mathbf{C N}), 46.25$ $(\mathbf{C H P h}), 46.10\left(\mathrm{C}(\mathrm{O}) \mathbf{C H}_{2}\right), 43.52\left(\mathbf{C}(\mathrm{CN})_{2}\right), 40.73\left(\mathbf{C H}_{2} \mathrm{CH}=\right), 30.86\left(\mathrm{CH}_{3}\right)$. The assignments of the ${ }^{1} \mathrm{H}$ and ${ }^{13} \mathrm{C}$ were based on DEPT, COSY, HMQC etc. 

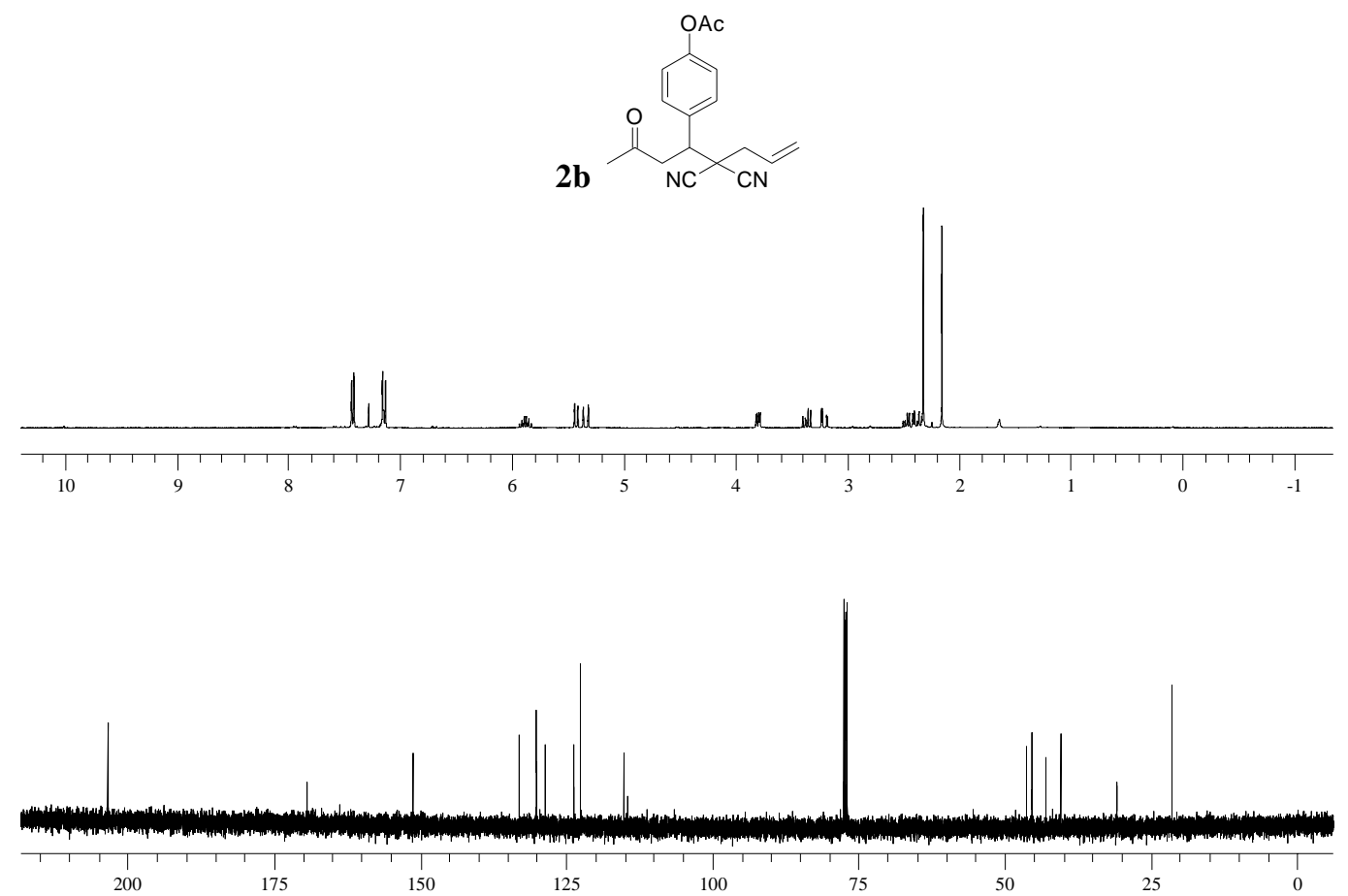

2b: Oil: ${ }^{1} \mathrm{H}$ NMR $\left(400 \mathrm{MHz}, \mathrm{CDCl}_{3}\right) \delta 7.42$ (m, $J=8.6,2 \mathrm{H}$ : arom H), $7.14(\mathrm{~m}, J=8.6,2 \mathrm{H}$ : arom H) 5.93-5.83 (m, 1H, $J=10.1,17.4,7.6,5.8 \mathrm{~Hz}:=\mathrm{CH}), 5.43\left(\mathrm{~d}, 1 \mathrm{H}, J=10.1 \mathrm{~Hz}: \mathrm{CH}=\mathrm{CH}(H)_{\mathrm{E}}\right), 5.34$ $\left(\mathrm{d}, 1 \mathrm{H}, J=17.4 \mathrm{~Hz}: \mathrm{CH}=\mathrm{CH}(H)_{\mathrm{Z}}\right), 3.80\left(\mathrm{dd}, 1 \mathrm{H}, J=3.5,9.8 \mathrm{~Hz}: \mathrm{C}(\mathrm{O}) \mathrm{CH}_{2} \mathrm{CH}\right), 3.36$ (dd, $J=9.8$, $\left.17.7 \mathrm{~Hz}, 1 \mathrm{H}: \mathrm{C}(\mathrm{O}) \mathrm{CH}_{2}\right), 3.20\left(\mathrm{dd}, J=3.5,17.7 \mathrm{~Hz}, 1 \mathrm{H}: \mathrm{C}(\mathrm{O}) \mathrm{CH}_{2}\right), 2.47(\mathrm{dd}, J=7.6,13.9 \mathrm{~Hz}, 1 \mathrm{H}$ : $\left.\mathrm{CH}_{2} \mathrm{CH}=\right), 2.39\left(\mathrm{dd}, J=5.8,13.9 \mathrm{~Hz}, 1 \mathrm{H}: \mathrm{CH}_{2} \mathrm{CH}=\right)$, 2.32(s, $\left.3 \mathrm{H}: \mathrm{OC}(\mathrm{O}) \mathrm{CH}_{3}\right), 2.15(\mathrm{~s}, 3 \mathrm{H}:$ $\left.\mathrm{C}(\mathrm{O}) \mathrm{CH}_{3}\right) ;{ }^{13} \mathrm{C} \mathrm{NMR}\left(100 \mathrm{MHz}, \mathrm{CDCl}_{3}\right) \delta 203.40(\mathrm{C}=\mathrm{O}), 169.27(\mathrm{OC}=\mathrm{O}), 151.24$ (Quat.), 132.95 (Quat.), 130.05 (Arom. $\mathbf{C H}), 128.56(=\mathbf{C H}), 123.66\left(=\mathrm{CH}_{2}\right), 122.53$ (Arom. $\mathbf{C H}$ ), 115.17 $(\mathbf{C N}), 114.44(\mathbf{C N}), 46.32\left(\mathrm{C}(\mathrm{O}) \mathbf{C H}_{2}\right), 45.30(\mathbf{C H A r}), 42.98\left(\mathbf{C}(\mathrm{CN})_{2}\right), 40.37\left(\mathbf{C H}_{2} \mathrm{CH}=\right), 30.75$ $\left(\mathrm{CH}_{3}\right), 21.35\left(\mathrm{OC}(\mathrm{O}) \mathbf{C H}_{3}\right)$. IR (CH2Cl2): vmax 3062,2252,1770,1724,1425, 989, 914. HRMS calcd for $\mathrm{C} 18 \mathrm{H} 18 \mathrm{~N} 2 \mathrm{O} 3 \mathrm{Na} 1$ [M+Na] 333.1215, found 333.1208.

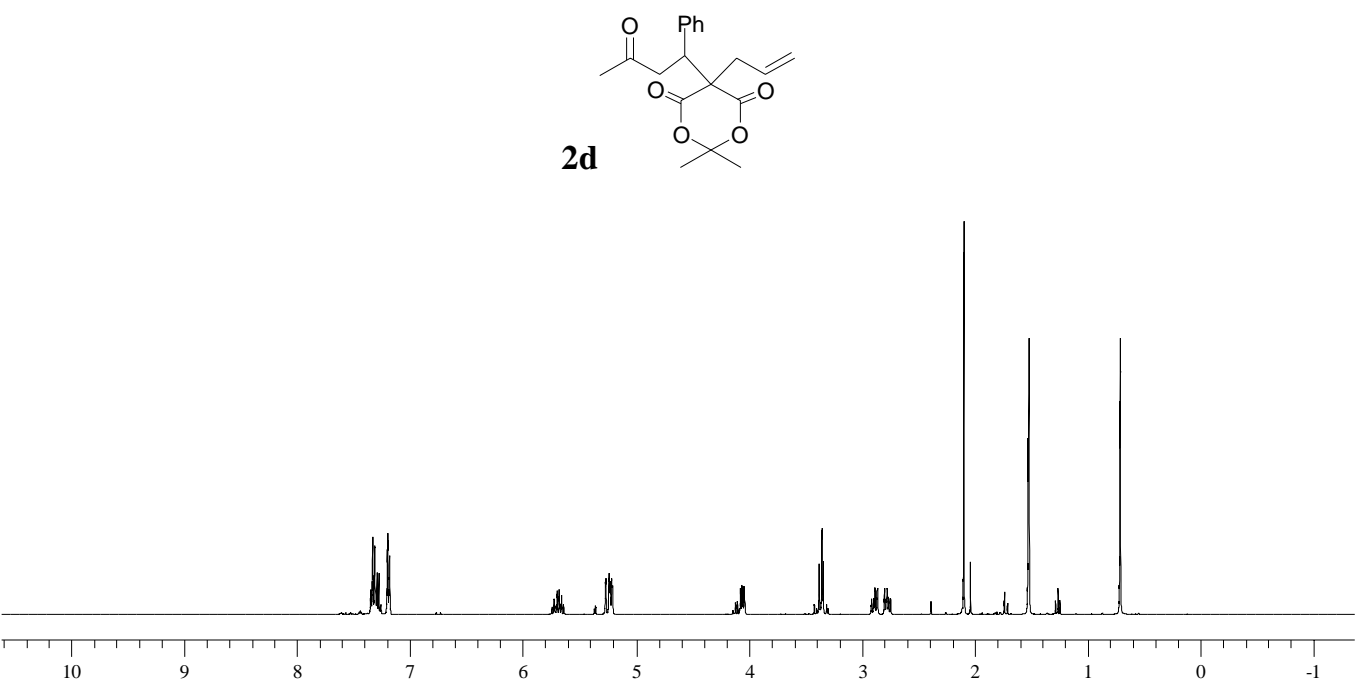




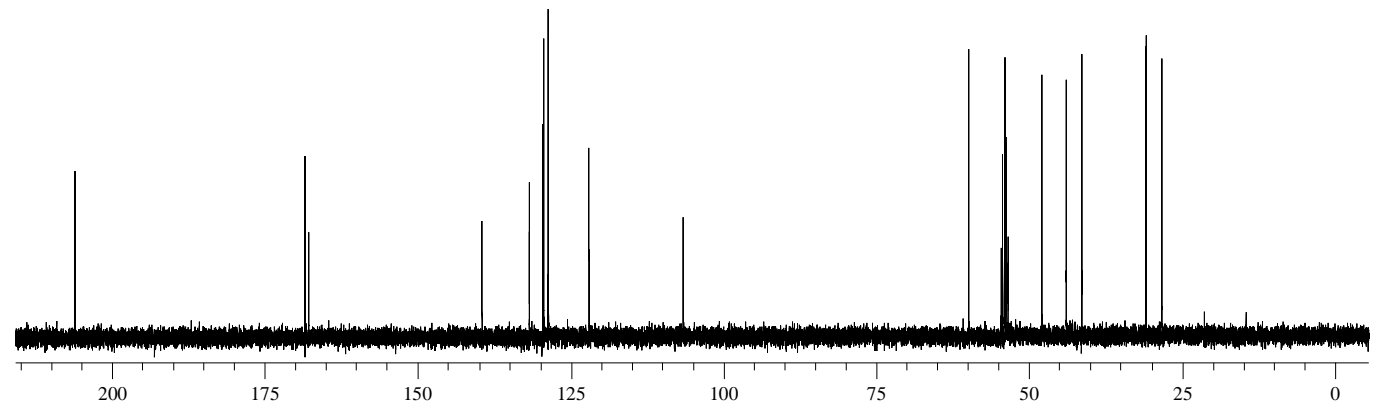

2d: Oil: ${ }^{1} \mathrm{H}$ NMR $\left(400 \mathrm{MHz}, \mathrm{CD}_{2} \mathrm{Cl}_{2}\right) \delta 7.31(\mathrm{~m}, 3 \mathrm{H}$ : arom $\mathrm{H}), \delta 7.19(\mathrm{~d}, J=6.8 \mathrm{~Hz}, 2 \mathrm{H}$ : arom H), 5.74-5.64 (m, $1 \mathrm{H}, J=12.9,5.1,12.6 \mathrm{~Hz}:=\mathrm{CH}), 5.25\left(\mathrm{~d}, 1 \mathrm{H}, J=12.9 \mathrm{~Hz}: \mathrm{CH}=\mathrm{CH}(H)_{\mathrm{z}}\right), 5.22(\mathrm{~d}$, $\left.1 \mathrm{H}, J=5.1 \mathrm{~Hz}: \mathrm{CH}=\mathrm{CH}(H)_{\mathrm{E}}\right), 4.05\left(\mathrm{dd}, 1 \mathrm{H}, J=8.8,5.3 \mathrm{~Hz}: \mathrm{C}(\mathrm{O}) \mathrm{CH}_{2} \mathrm{CHPh}\right), 3.35$ (app t, $2 \mathrm{H}$ : $\left.\mathrm{C}(\mathrm{O}) \mathrm{CH}_{2}\right), 2.89$ (dd, $\left.J=7.8,12.6 \mathrm{~Hz}, 1 \mathrm{H}: \mathrm{CH}_{2} \mathrm{CH}=\right), 2.77$ (dd, $J=7.3,12.6 \mathrm{~Hz}, 1 \mathrm{H}: \mathrm{CH}_{2} \mathrm{CH}=$ ), 2.10 (s, 3H: $\left.\mathrm{C}(\mathrm{O}) \mathrm{CH}_{3}\right), 1.52$ (s, 3H: $\left.\mathrm{CH}_{3}\right), 0.71$ (s, 3H: $\left.\mathrm{CH}_{3}\right) ;{ }^{13} \mathrm{C} \mathrm{NMR}\left(100 \mathrm{MHz}, \mathrm{CD}_{2} \mathrm{Cl}_{2}\right) \delta$ $206.01(\mathbf{C}=\mathrm{O}), 168.46(\mathrm{OC}=\mathrm{O}) 167.80(\mathrm{OC}=\mathrm{O}) 139.41$ (Quat.), $131.70(=\mathbf{C H}), 129.59$ (Arom. $\mathbf{C H}), 129.38$ (Arom. $\mathbf{C H}), 128.57$ (Arom. $\mathbf{C H}), 121.99\left(=\mathrm{CH}_{2}\right), 106.64\left(\mathbf{C}\left(\mathrm{CH}_{3}\right)_{2}\right), 59.89$ $\left(\mathbf{C}\left(\mathrm{CO}_{2} \mathrm{R}\right)_{2}\right), 47.89(\mathbf{C H P h}), 43.91 \quad\left(\mathrm{C}(\mathrm{O}) \mathbf{C H}_{2}\right), 41.42 \quad\left(\mathbf{C H}_{2} \mathbf{C H}=\right), 30.91 / 30.82$ (overlapping $\mathrm{C}(\mathrm{O}) \mathrm{CH}_{3}$ with $\left.\mathrm{CH}_{3}\right), 28.26\left(\mathrm{CH}_{3}\right)$. IR $(\mathrm{CH} 2 \mathrm{Cl} 2)$ : vmax 3063,1765,1732,1456. HRMS calcd for C19H22O5Na1 [M+Na] 353.1365, found 353.1347.

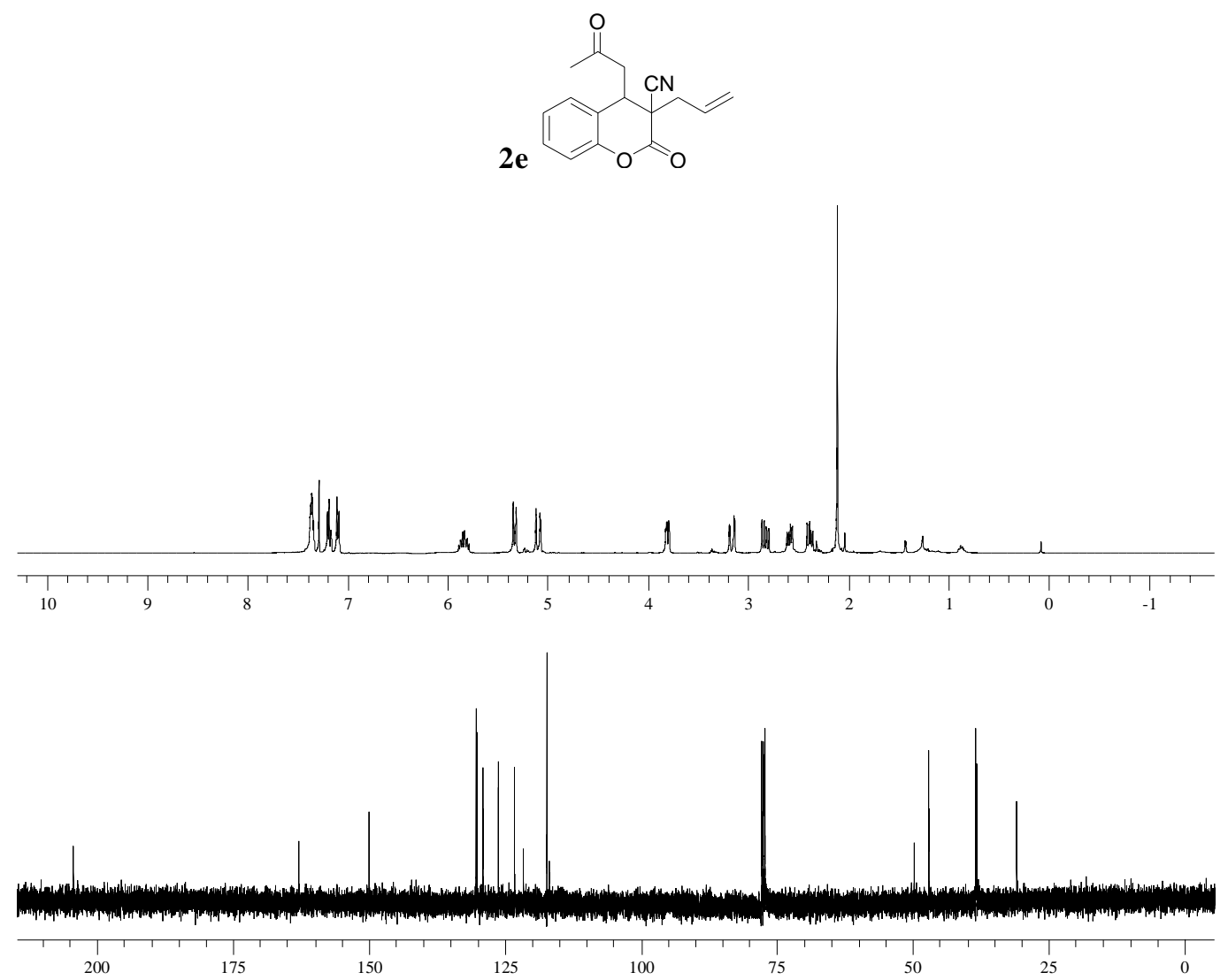

2e: Oil: ${ }^{1} \mathrm{H}$ NMR (500 MHz, $\mathrm{CDCl}_{3}$ ) Major Diastereomer: $\delta 7.35$ (m, 2H: arom H), $\delta 7.18$ (app. t, 1H: arom H), $\delta 7.09$ (app. d, 1H: arom H), $\delta 5.85(\mathrm{~m}, 1 \mathrm{H}, J=10.1,16.9 \mathrm{~Hz}:=\mathrm{CH}), 5.33(\mathrm{~d}, 1 \mathrm{H}, J=$ 
$\left.10.1 \mathrm{~Hz}, \mathrm{CH}=\mathrm{CH}(H)_{\mathrm{E}}\right), 5.10\left(\mathrm{~d}, 1 \mathrm{H}, J=16.9 \mathrm{~Hz}: \mathrm{CH}=\mathrm{CH}(H)_{\mathrm{Z}}\right), 3.80(\mathrm{dd}, 1 \mathrm{H}, J=6.6,3.0 \mathrm{~Hz}: \mathrm{CH})$, $3.16\left(\mathrm{dd}, 1 \mathrm{H}, J=17.9,2.8 \mathrm{~Hz}: \mathrm{C}(\mathrm{O}) \mathrm{CH}_{2}\right), 2.83\left(\mathrm{dd}, 1 \mathrm{H}, J=17.9,9.6 \mathrm{~Hz},: \mathrm{C}(\mathrm{O}) \mathrm{C} \mathbf{H}_{2}\right), 2.59(\mathrm{dd}, J$ $\left.=6.6,14.1 \mathrm{~Hz}, 1 \mathrm{H}: \mathrm{CH}_{2} \mathrm{CH}=\right), 2.38\left(\mathrm{dd}, 1 \mathrm{H}, J=8.1,13.9 \mathrm{~Hz}: \mathrm{CH}_{2} \mathrm{CH}=\right), 2.11\left(3 \mathrm{H}: \mathrm{C}(\mathrm{O}) \mathrm{CH}_{3}\right) ;{ }^{13} \mathrm{C}$ NMR (100 MHz, $\left.\mathrm{CDCl}_{3}\right) \delta 206.35(\mathbf{C}=\mathrm{O}), 162.95$ (OC=O), 149.94 (Quat.), 130.31 (Arom. $\mathbf{C H}$ ), $129.04(=\mathbf{C H}), 126.11$ (Arom. $\mathbf{C H}), 123.16\left(=\mathrm{CH}_{2}\right), 121.51$ (Quat.), 117.25 (Arom. $\left.\mathbf{C H}\right), 116.84$ $(\mathbf{C N}), 49.70\left(\mathrm{t}-\mathbf{C}(\mathrm{CN})\left(\mathrm{CO}_{2}\right)\right), 47.03\left(\mathrm{C}(\mathrm{O}) \mathbf{C H}_{2}\right), 38.38$ (overlapping $\mathbf{C H}_{2} \mathbf{C H}=, \mathbf{C H C H}_{2} \mathrm{CO}$ ), 30.83 $\left(\mathrm{CH}_{3}\right)$. IR (CH3O $\left.{ }^{\mathrm{B} u}\right): v \max 2250,1780,1726,912$. HRMS calcd for $\mathrm{C} 16 \mathrm{H} 15 \mathrm{NO} 3$ [M+Na] 292.0950, found [M+Na] 292.0946.
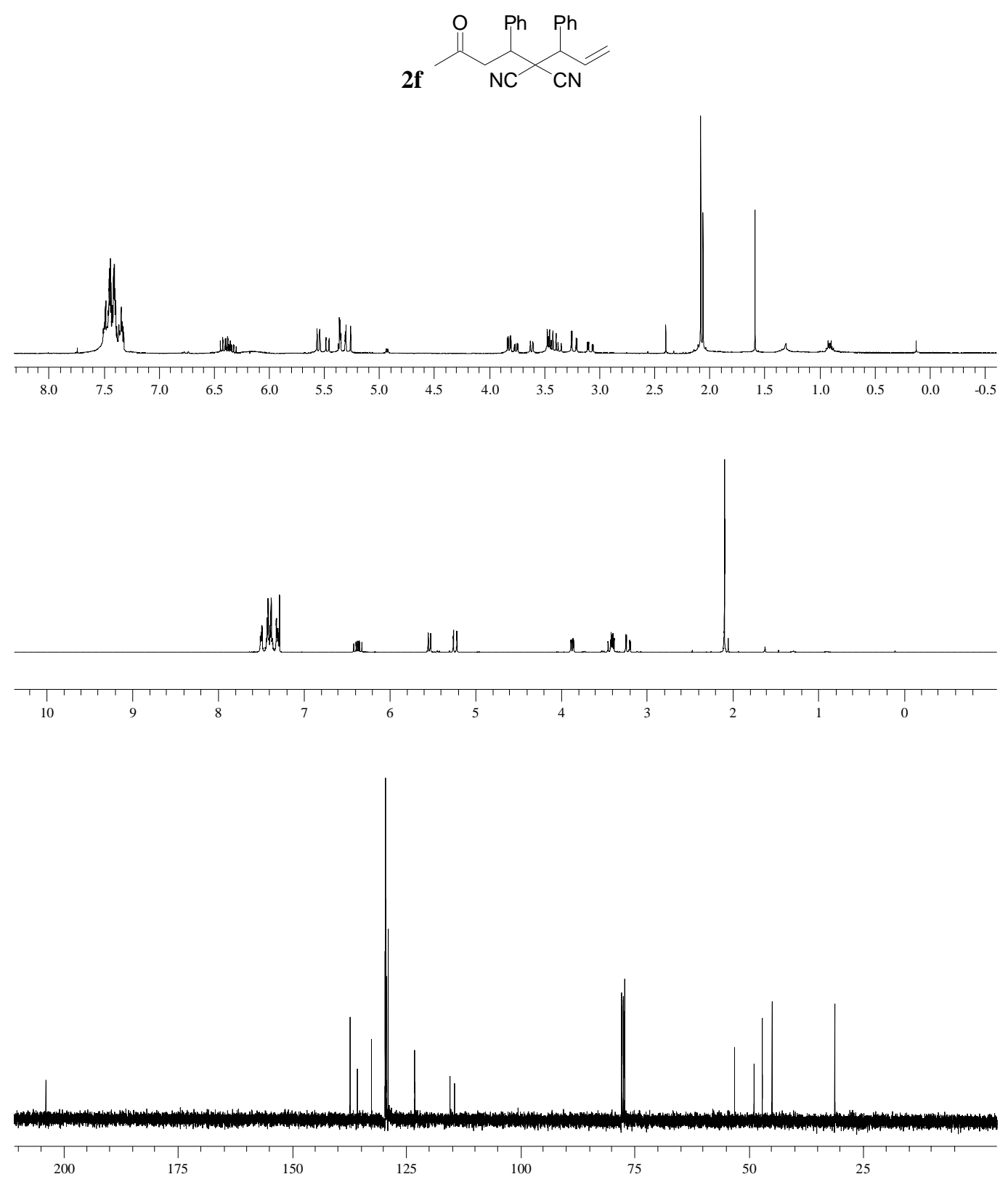

2f: Oil: ${ }^{1} \mathrm{H}$ NMR $\left(400 \mathrm{MHz}, \mathrm{CDCl}_{3}\right)$ Major diastereomer: $\delta$ 7.50-7.28 (m, 10H: arom H), 6.41-6.32 $(\mathrm{m}, 1 \mathrm{H}, J=16.9,10.4,9.1 \mathrm{~Hz}:=\mathrm{CH}), 5.53\left(\mathrm{~d}, 1 \mathrm{H}, J=10.4 \mathrm{~Hz}: \mathrm{CH}=\mathrm{CH}(H)_{\mathrm{E}}\right), 5.23(\mathrm{~d}, 1 \mathrm{H}, J=16.9$ $\left.\mathrm{Hz}: \mathrm{CH}=\mathrm{CH}(H)_{\mathrm{z}}\right), 3.87\left(\mathrm{dd}, 1 \mathrm{H}, J=10.6,3.3 \mathrm{~Hz}: \mathrm{C}(\mathrm{O}) \mathrm{CH}_{2} \mathrm{CHPh}\right.$ ), 3.45-3.38 (m, 2H: overlapping 
CHPh, $\left.\mathrm{C}(\mathrm{O}) \mathrm{CH}_{2}\right), 3.22\left(\mathrm{dd}, J=17.4,3.3 \mathrm{~Hz}, 1 \mathrm{H}: \mathrm{C}(\mathrm{O}) \mathrm{C} \mathbf{H}_{2}\right), 2.09$ (s, 3H: $\left.\mathrm{C}(\mathrm{O}) \mathrm{C} \mathbf{H}_{3}\right) ;{ }^{13} \mathrm{C}$ NMR $(100$ $\mathrm{MHz}, \mathrm{CDCl}_{3}$ ) Major isomer: $\delta 203.76(\mathbf{C}=\mathrm{O}), 137.10$ (Quat.), 135.52 (Quat.), $132.40(=\mathbf{C H})$, 129.34 (Arom. CH), 129.35 (Arom. CH), 128.73 (Arom. $\mathbf{C H}), 122.90\left(=\mathrm{CH}_{2}\right), 115.13(\mathbf{C N})$, $114.21(\mathbf{C N}), 52.90(\mathbf{C H P h}), 48.65\left(\mathbf{C}(\mathrm{CN})_{2}\right), 46.85\left(\mathrm{C}(\mathrm{O}) \mathbf{C H}_{2}\right), 44.68\left(\mathrm{C}(\mathrm{O}) \mathrm{CH}_{2} \mathbf{C H P h}\right), 30.94$ $\left(\mathrm{CH}_{3}\right)$. IR (CH2Cl2): vmax 3053,2304,1724,1421, 991, 895. HRMS calcd for $\mathrm{C} 22 \mathrm{H} 20 \mathrm{~N} 2 \mathrm{O} 1 \mathrm{Na} 1$ [M+Na] 351.1473, found 351.1468.

Minor diastereomer: $\delta$ 7.50-7.28 (m, $10 \mathrm{H}$ : arom $\mathrm{H}$, overlapping minor/major isomers), 6.44-6.30 (m, 1H, overlapping minor/major isomer: $=\mathrm{CH}), 5.47\left(\mathrm{~d}, 1 \mathrm{H}, J=10.1 \mathrm{~Hz}: \mathrm{CH}=\mathrm{CH}(H)_{\mathrm{E}}\right)$, 5.36-5.26 (d, 1H, overlapping minor/major isomer: $\left.\mathrm{CH}=\mathrm{CH}(H)_{\mathrm{Z}}\right), 3.76(\mathrm{dd}, 1 \mathrm{H}, J=10.6,3.0 \mathrm{~Hz}$ : $\mathrm{C}(\mathrm{O}) \mathrm{CH}_{2} \mathrm{CHPh}$ ), 3.45-3.38 (m, 2H: overlapping $\left.\mathrm{CHPh}, \mathrm{C}(\mathrm{O}) \mathrm{CH}_{2}\right), 3.08$ (dd, $J=17.2,3.0 \mathrm{~Hz}, 1 \mathrm{H}$ : $\mathrm{C}(\mathrm{O}) \mathrm{CH}_{2}$ ), 2.06(s, 3H: $\left.\mathrm{C}(\mathrm{O}) \mathrm{CH}_{3}\right) ;{ }^{13} \mathrm{C}$ NMR $\left(100 \mathrm{MHz}, \mathrm{CDCl}_{3}\right.$ ): $\delta 203.76$ (overlapping $\mathbf{C}=\mathrm{O}$ ), 136.65 (Quat.), 135.20 (Quat.), 133.84 (=CH), 129.58-128.91 (overlapping minor/major isomer: Arom. $\mathbf{C H}), 121.88\left(=\mathrm{CH}_{2}\right), 115.09(\mathbf{C N}), 114.63(\mathbf{C N}), 53.09(\mathbf{C H P h}), 48.84\left(\mathbf{C}(\mathrm{CN})_{2}\right), 45.76$ $\left(\mathrm{C}(\mathrm{O}) \mathrm{CH}_{2}\right), 44.71\left(\mathrm{C}(\mathrm{O}) \mathrm{CH}_{2} \mathbf{C H P h}\right), 30.85\left(\mathrm{CH}_{3}\right)$.
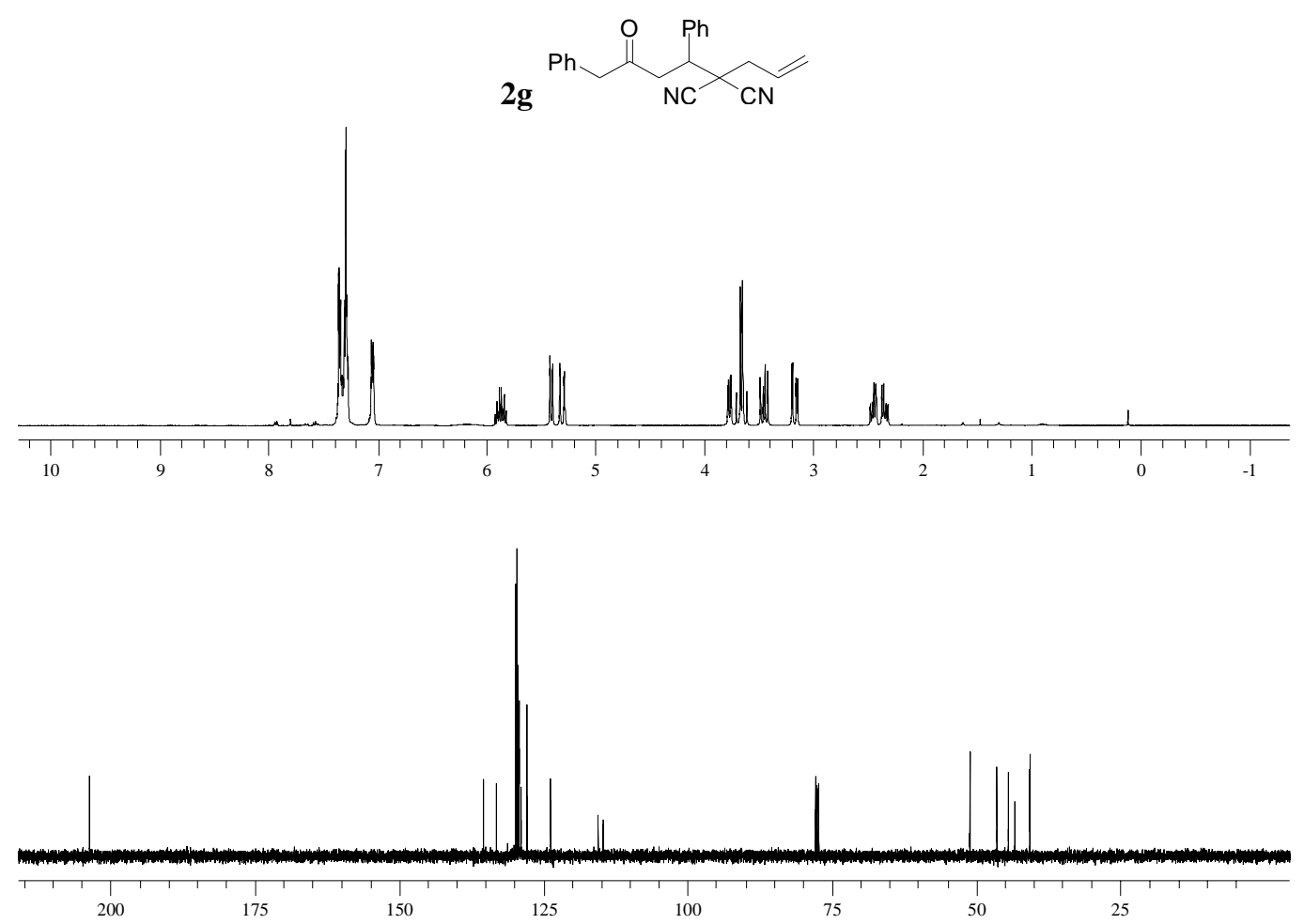

2g: Oil: ${ }^{1} \mathrm{H}$ NMR (400 MHz, $\left.\mathrm{CDCl}_{3}\right) \delta 7.32(\mathrm{~m}, 8 \mathrm{H}$ : arom H), 7.05 (m, 2H: arom H), $5.87(\mathrm{~m}, 1 \mathrm{H}, J$ $=10.1,16.9,7.6,7.1 \mathrm{~Hz}:=\mathrm{CH}), 5.41\left(\mathrm{~d}, 1 \mathrm{H}, J=10.1 \mathrm{~Hz}: \mathrm{CH}=\mathrm{CH}(H)_{\mathrm{E}}\right), 5.31(\mathrm{~d}, 1 \mathrm{H}, J=16.9 \mathrm{~Hz}$ : $\left.\mathrm{CH}=\mathrm{CH}(H)_{\mathrm{Z}}\right), 3.77\left(\mathrm{dd}, 1 \mathrm{H}, J=10.4,3.3 \mathrm{~Hz}: \mathrm{C}(\mathrm{O}) \mathrm{CH}_{2} \mathrm{CHPh}\right), 3.66\left(\mathrm{AB}, 2 \mathrm{H}: \mathrm{PhCH}_{2} \mathrm{C}(\mathrm{O})\right), 3.45$ $\left(\mathrm{dd}, J=10.6,17.4 \mathrm{~Hz}, 1 \mathrm{H}: \mathrm{C}(\mathrm{O}) \mathrm{CH}_{2}\right), 3.22\left(\mathrm{dd}, J=3.5,17.2 \mathrm{~Hz}, 1 \mathrm{H}: \mathrm{C}(\mathrm{O}) \mathrm{CH}_{2}\right), 2.46(\mathrm{dd}, J=7.6$, $\left.14.1 \mathrm{~Hz}, 1 \mathrm{H}: \mathrm{CH}_{2} \mathrm{CH}=\right), 2.42\left(\mathrm{dd}, J=7.1,13.9 \mathrm{~Hz}, 1 \mathrm{H}: \mathrm{CH}_{2} \mathrm{CH}=\right) ;{ }^{13} \mathrm{C} \mathrm{NMR}\left(100 \mathrm{MHz}, \mathrm{CD}_{2} \mathrm{Cl}_{2}\right)$

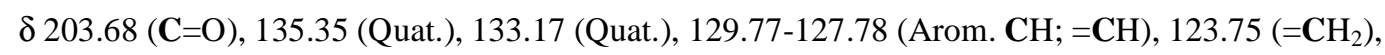
$115.46(\mathbf{C N}), 114.67(\mathbf{C N}), 51.03\left(\mathrm{PhCH}_{2} \mathrm{C}(\mathrm{O})\right), 46.37(\mathbf{C H P h}), 44.35\left(\mathrm{C}(\mathrm{O}) \mathbf{C H}_{2}\right), 43.23\left(\mathbf{C}(\mathrm{CN})_{2}\right)$, $40.68\left(\mathrm{CH}_{2} \mathrm{CH}=\right)$. IR $(\mathrm{CH} 2 \mathrm{Cl} 2)$ : vmax 2251,1720, 1454, 989. HRMS calcd for $\mathrm{C} 22 \mathrm{H} 20 \mathrm{~N} 2 \mathrm{O} 1 \mathrm{Na} 1$ [M+Na] 351.1473, found 351.1481. 


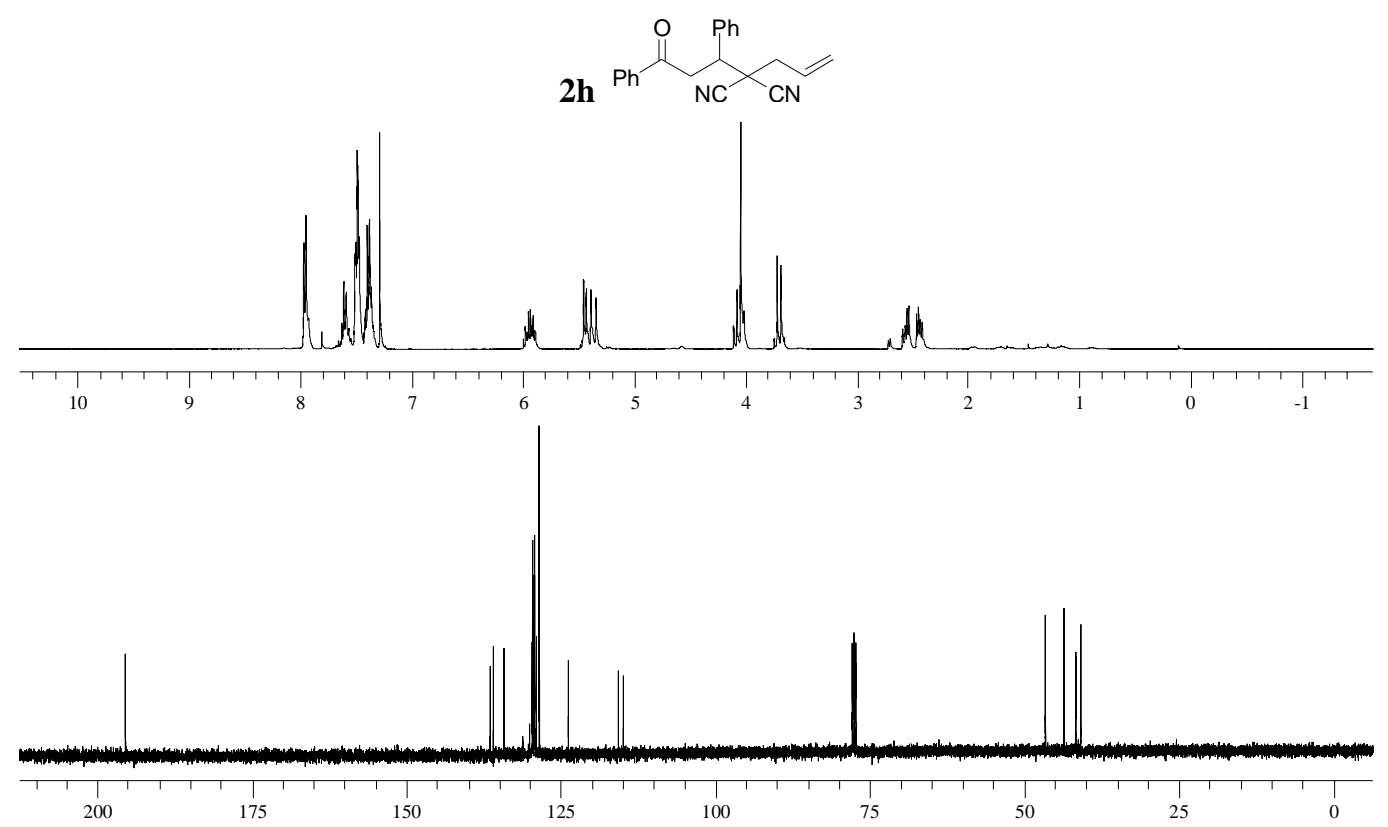

2h: Oil: ${ }^{1} \mathrm{H}$ NMR (400 MHz, $\left.\mathrm{CDCl}_{3}\right) \delta$ 7.96-7.38 (10H: arom H), $5.95(\mathrm{~m}, 1 \mathrm{H}, J=10.1,16.9,7.1$, $7.3 \mathrm{~Hz}:=\mathrm{CH}), 5.44\left(\mathrm{~d}, 1 \mathrm{H}, J=10.1 \mathrm{~Hz}: \mathrm{CH}=\mathrm{CH}(H)_{\mathrm{E}}\right), 5.36\left(\mathrm{~d}, 1 \mathrm{H}, J=16.9 \mathrm{~Hz}: \mathrm{CH}=\mathrm{CH}(H)_{\mathrm{Z}}\right)$, 4.10-4.01 (m, 2H: overlapping $\left.\mathrm{C}(\mathrm{O}) \mathrm{CH}_{2} \mathrm{CHPh}, \mathrm{C}(\mathrm{O}) \mathrm{CH}_{2}\right), 3.70\left(\mathrm{~d}, 1 \mathrm{H}, J=14.4 \mathrm{~Hz}: \mathrm{C}(\mathrm{O}) \mathrm{CH}_{2}\right)$, 2.55 (dd, $\left.J=7.3,13.9 \mathrm{~Hz}, 1 \mathrm{H}: \mathrm{CH}_{2} \mathrm{CH}=\right), 2.43\left(\mathrm{dd}, J=7.1,13.9 \mathrm{~Hz}, 1 \mathrm{H}: \mathrm{CH}_{2} \mathrm{CH}=\right)$; ${ }^{13} \mathrm{C} \mathrm{NMR}$ (100 MHz, $\mathrm{CD}_{2} \mathrm{Cl}_{2}$ ) $\delta 195.46$ (C=O), 136.46 (Quat.), 135.92 (Quat.), 129.57-128.51 (overlapping Arom. $\mathbf{C H} ;=\mathbf{C H}), 123.80\left(=\mathrm{CH}_{2}\right), 115.69(\mathbf{C N}), 114.92(\mathbf{C N}), 46.53(\mathbf{C H P h}), 43.53\left(\mathbf{C}(\mathrm{CN})_{2}\right)$, $41.66\left(\mathrm{C}(\mathrm{O}) \mathrm{CH}_{2}\right), 40.83\left(\mathrm{CH}_{2} \mathrm{CH}=\right)$. IR $(\mathrm{CH} 2 \mathrm{Cl} 2)$ : vmax 3053,2308, 1689,1421, 989, 895. HRMS calcd for $\mathrm{C} 21 \mathrm{H} 19 \mathrm{~N} 2 \mathrm{O} 1[\mathrm{M}+\mathrm{H}] 315.1497$, found 315.1493 .
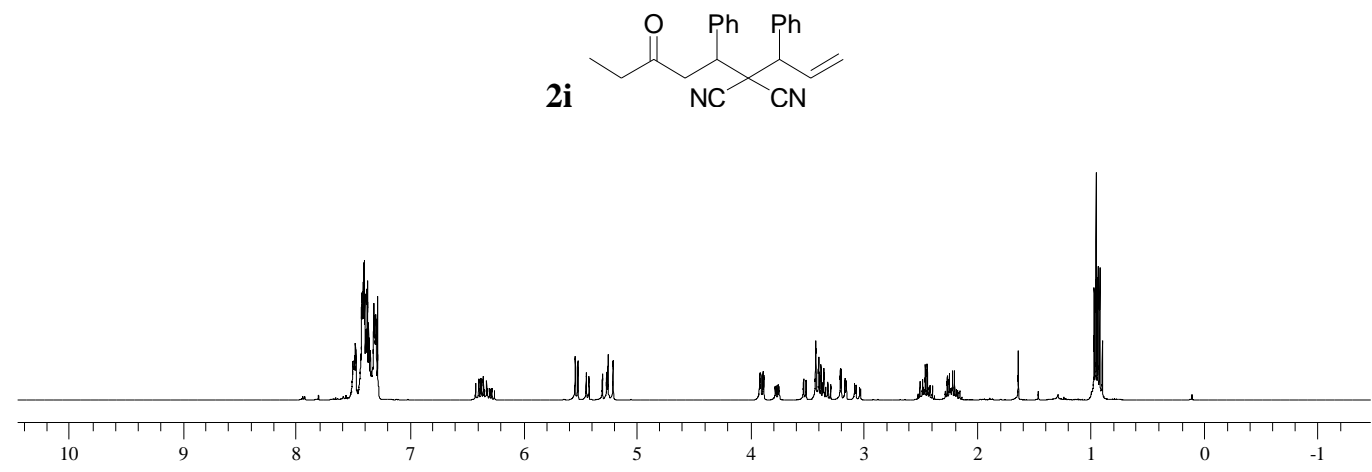


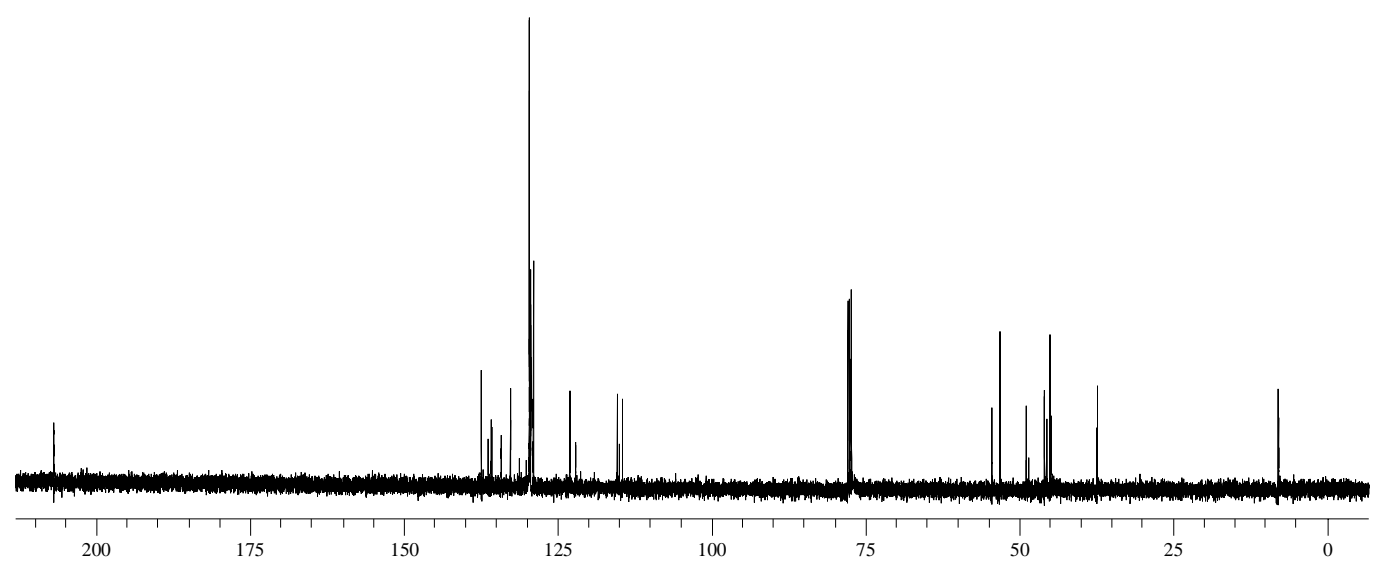

2i: Oil: ${ }^{1} \mathrm{H}$ NMR (400 MHz, $\mathrm{CDCl}_{3}$ ) Major Diastereomer: $\delta$ 7.50-7.28 (m, $10 \mathrm{H}$ : arom $\mathrm{H}$ ), 6.42-6.33 $(\mathrm{m}, 1 \mathrm{H}, J=16.9,10.4, \mathrm{~Hz}:=\mathrm{CH}), 5.53\left(\mathrm{~d}, 1 \mathrm{H}, J=10.4 \mathrm{~Hz}: \mathrm{CH}=\mathrm{CH}(H)_{\mathrm{E}}\right), 5.23(\mathrm{~d}, 1 \mathrm{H}, J=16.9 \mathrm{~Hz}$ : $\left.\mathrm{CH}=\mathrm{CH}(H)_{\mathrm{Z}}\right), 3.90\left(\mathrm{dd}, 1 \mathrm{H}, J=10.6,3.3 \mathrm{~Hz}: \mathrm{C}(\mathrm{O}) \mathrm{CH}_{2} \mathrm{CHPh}\right), 3.53-3.29(\mathrm{~m}, 2 \mathrm{H}$ : overlapping $\left.\mathrm{CHPh}, \mathrm{C}(\mathrm{O}) \mathrm{CH}_{2}\right), 3.18$ (dd, $\left.J=13.9,3.3 \mathrm{~Hz}, 1 \mathrm{H}: \mathrm{C}(\mathrm{O}) \mathrm{CH}_{2}\right), 2.45$ (m, 1H: $\left.\mathrm{C}(\mathrm{O}) \mathrm{CH} \mathbf{H}_{2}\right), 2.21(\mathrm{~m}, 1 \mathrm{H}$ : $\mathrm{C}(\mathrm{O}) \mathrm{CH}_{2}$ ), 0.95(app. t, 3H: C(O)CH $\left.\mathrm{CH}_{2} \mathrm{CH}_{3}\right) ;{ }^{13} \mathrm{C}$ NMR (100 MHz, $\left.\mathrm{CDCl}_{3}\right) \delta 206.84(\mathbf{C}=\mathrm{O}), 137.30$ (Quat.), 135.61 (Quat.), $132.61(=\mathbf{C H}), 129.51-128.92$ (Arom. $\mathbf{C H}), 123.30\left(=\mathbf{C H}_{2}\right), 115.32(\mathbf{C N})$, $114.42(\mathbf{C N}), 53.09(\mathbf{C H P h}), 48.84\left(\mathbf{C}(\mathrm{CN})_{2}\right), 45.85\left(\mathrm{C}(\mathrm{O}) \mathbf{C H}_{2}\right), 44.94\left(\mathrm{C}(\mathrm{O}) \mathrm{CH}_{2} \mathbf{C H P h}\right), 37.22$ $\left(\mathrm{CH}_{3} \mathbf{C H}_{2}\right), 7.82\left(\mathrm{CH}_{3}\right)$. IR $(\mathrm{CH} 2 \mathrm{Cl} 2)$ : vmax 2252, 1720,1456, 991. HRMS calcd for $\mathrm{C} 23 \mathrm{H} 22 \mathrm{~N} 2 \mathrm{O} 1 \mathrm{Na} 1 \mathrm{M}+\mathrm{Na}] 365.2$, found 365.1.

Minor diastereomer: $\delta$ 7.50-7.28 (m, $10 \mathrm{H}$, arom $\mathrm{H}$ : overlapping minor/major isomer), 6.34-6.25 (m, 1H, =CH: overlapping minor/major isomer), $5.43\left(\mathrm{~d}, 1 \mathrm{H}, J=10.4 \mathrm{~Hz}: \mathrm{CH}=\mathrm{CH}(H)_{\mathrm{E}}\right)$, $5.28\left(\mathrm{~d}, 1 \mathrm{H}, J=16.9 \mathrm{~Hz}: \mathrm{CH}=\mathrm{CH}(H)_{\mathrm{z}}\right), 3.76\left(\mathrm{dd}, 1 \mathrm{H}, J=10.6,3.3 \mathrm{~Hz}: \mathrm{C}(\mathrm{O}) \mathrm{CH}_{2} \mathrm{CHPh}\right), 3.53-3.29$ $\left(\mathrm{m}, 2 \mathrm{H}\right.$ : overlapping minor/major isomer $\left.\mathrm{CHPh}, \mathrm{C}(\mathrm{O}) \mathrm{CH}_{2}\right), 3.05(\mathrm{dd}, J=13.9,3.3 \mathrm{~Hz}, 1 \mathrm{H}$ : $\left.\mathrm{C}(\mathrm{O}) \mathrm{CH}_{2}\right), 2.45\left(\mathrm{~m}, 1 \mathrm{H}\right.$ : overlapping minor/major isomer $\left.\mathrm{C}(\mathrm{O}) \mathrm{CH}_{2}\right), 2.21(\mathrm{~m}, 1 \mathrm{H}$ : overlapping minor/major isomer $\mathrm{C}(\mathrm{O}) \mathrm{CH}_{2}$ ), 0.95(app. t, 3H: overlapping minor/major isomer $\left.\mathrm{C}(\mathrm{O}) \mathrm{CH}_{2} \mathrm{CH}_{3}\right)$; ${ }^{13} \mathrm{C}$ NMR (100 MHz, $\left.\mathrm{CDCl}_{3}\right) \delta 206.84(\mathbf{C}=\mathrm{O}$ : overlapping minor/major isomer), 136.22 (Quat.), 135.61 (Quat.), 134.09 (=CH), 129.51-128.92 (Arom. $\mathbf{C H}), 121.99\left(=\mathbf{C H}_{2}\right), 114.89(\mathbf{C N}), 114.42$ $(\mathbf{C N}), 54.35(\mathbf{C H P h}), 48.35\left(\mathbf{C}(\mathrm{CN})_{2}\right), 45.47\left(\mathrm{C}(\mathrm{O}) \mathbf{C H}_{2}\right), 44.65\left(\mathrm{C}(\mathrm{O}) \mathrm{CH}_{2} \mathbf{C H P h}\right), 37.29\left(\mathrm{CH}_{3} \mathbf{C H}_{2}\right)$, 7. $77\left(\mathrm{CH}_{3}\right)$.
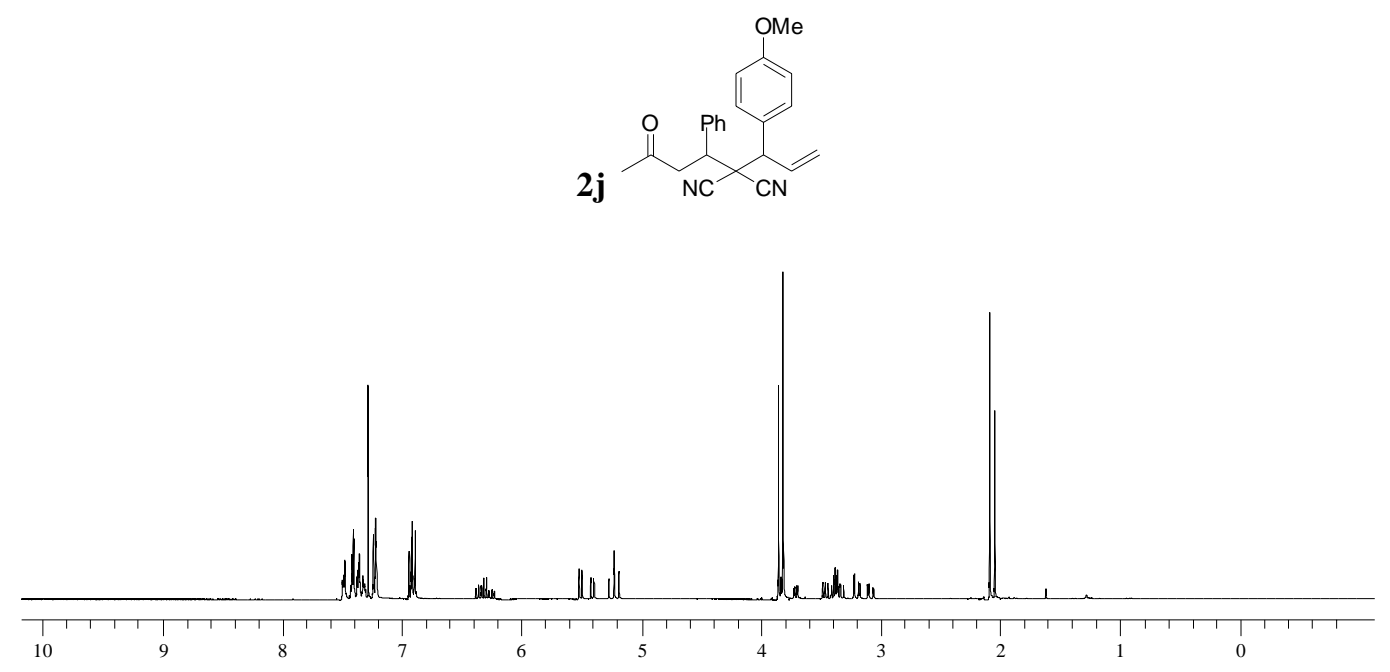


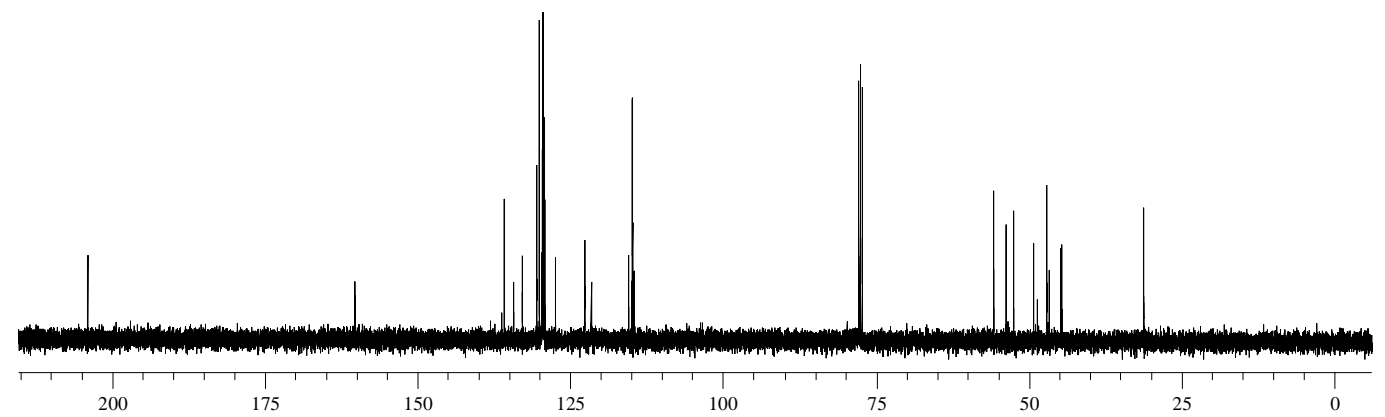

2j: Oil: ${ }^{1} \mathrm{H}$ NMR (400 MHz, $\mathrm{CDCl}_{3}$ ) Major Diastereomer: $\delta$ 7.50-7.21 (m, 5H: arom H), 6.94-6.88 (m, 4H: Arom. H), 6.38-6.23 (m, 1H, $J=16.9,10.1,9.1 \mathrm{~Hz}:=\mathrm{CH}), 5.51(\mathrm{~d}, 1 \mathrm{H}, J=10.1 \mathrm{~Hz}$ : $\left.\mathrm{CH}=\mathrm{CH}(H)_{\mathrm{E}}\right), 5.23\left(\mathrm{~d}, 1 \mathrm{H}, J=16.9 \mathrm{~Hz}: \mathrm{CH}=\mathrm{CH}(H)_{\mathrm{Z}}\right), 3.81\left(\mathrm{~s}, 3 \mathrm{H}: \mathrm{OCH}_{3}\right), 3.81-3.85(\mathrm{dd}, 1 \mathrm{H}$ : overlapping $\left.\mathrm{C}(\mathrm{O}) \mathrm{CH}_{2} \mathrm{CHPh}, \mathrm{OCH}_{3}\right), 3.48-3.31$ (m, 2H: overlapping $\mathrm{CHAr}, \mathrm{C}(\mathrm{O}) \mathrm{CH}$ ), 3.20 (dd, $J$ $\left.=17.2,3.0 \mathrm{~Hz}, 1 \mathrm{H}: \mathrm{C}(\mathrm{O}) \mathrm{CH}_{2}\right), 2.09$ (s, 3H: $\left.\mathrm{C}(\mathrm{O}) \mathrm{CH}_{3}\right) ;{ }^{13} \mathrm{C} \mathrm{NMR}\left(100 \mathrm{MHz}, \mathrm{CDCl}_{3}\right) \delta 203.96$ $(\mathbf{C}=\mathrm{O}), 160.27$ (Quat.), 135.74 (Quat.), $132.79(=\mathbf{C H}), 130.52-129.22$ (Arom. $\mathbf{C H}), 122.67\left(=\mathbf{C H}_{2}\right)$, 114.82 (Arom. $\mathbf{C H}), 115.38(\mathbf{C N}), 114.82(\mathbf{C N}), 55.73\left(\mathrm{OCH}_{3}\right), 52.40(\mathbf{C H A r}), 49.17\left(\mathbf{C}(\mathrm{CN})_{2}\right)$, $47.01\left(\mathrm{C}(\mathrm{O}) \mathrm{CH}_{2}\right), 44.74(\mathbf{C H P h}), 31.12\left(\mathrm{CH}_{3}\right) . \quad$ IR $(\mathrm{CH} 2 \mathrm{Cl} 2)$ : vmax 2247,1724,1253. HRMS calcd for $\mathrm{C} 23 \mathrm{H} 22 \mathrm{~N} 2 \mathrm{O} 7 \mathrm{Na} 1[\mathrm{M}+\mathrm{Na}] 381.1579$, found 381.1556.

Minor Diastereomer: $\delta$ 7.50-7.21 (m, 5H: arom $\mathrm{H}$, overlapping minor/major isomer), 6.94-6.88 (m, 4H: Arom. H, overlapping minor/major isomer), 6.38-6.23 (m, $1 \mathrm{H}:=\mathrm{CH}$, overlapping minor/major isomer), $5.41\left(\mathrm{~d}, 1 \mathrm{H}, J=10.1 \mathrm{~Hz}: \mathrm{CH}=\mathrm{CH}(H)_{\mathrm{E}}\right), 5.23(\mathrm{~d}, 1 \mathrm{H}$ : $\mathrm{CH}=\mathrm{CH}(H)_{\mathrm{Z}}$, overlapping minor/major isomer), 3.85 (s, $3 \mathrm{H}$ : $\left.\mathrm{OCH}_{3}\right), 3.71(\mathrm{dd}, 1 \mathrm{H}, J=10.9,3.0 \mathrm{~Hz}$ : $\mathrm{C}(\mathrm{O}) \mathrm{CH}_{2} \mathrm{CHPh}$ ), 3.48-3.31 (m, 2H: overlapping minor/major isomer: $\left.\mathrm{CHAr}, \mathrm{C}(\mathrm{O}) \mathrm{C} \mathbf{H}_{2}\right), 3.08$ (dd, $\left.J=17.2,3.0 \mathrm{~Hz}, 1 \mathrm{H}: \mathrm{C}(\mathrm{O}) \mathrm{CH}_{2}\right), 2.05\left(\mathrm{~s}, 3 \mathrm{H}: \mathrm{C}(\mathrm{O}) \mathrm{CH}_{3}\right) ;{ }^{13} \mathrm{C} \mathrm{NMR}\left(100 \mathrm{MHz}, \mathrm{CDCl}_{3}\right): \delta 203.96$ $(\mathbf{C}=\mathrm{O}$, overlapping minor/major isomer), 136.20 (Quat.), $134.26(=\mathbf{C H}), 130.52-129.22$ (Arom. $\mathbf{C H}), 127.41$ (Quat), $121.59\left(=\mathrm{CH}_{2}\right), 114.82$ (Arom. $\left.\mathbf{C H}\right), 114.94(\mathbf{C N}), 114.50(\mathbf{C N}), 55.67$ $\left(\mathrm{OCH}_{3}\right), 53.61(\mathbf{C H A r}), 48.58\left(\mathbf{C}(\mathrm{CN})_{2}\right), 46.58\left(\mathrm{C}(\mathrm{O}) \mathbf{C H}_{2}\right), 44.51(\mathbf{C H P h}), 31.17\left(\mathrm{CH}_{3}\right)$.

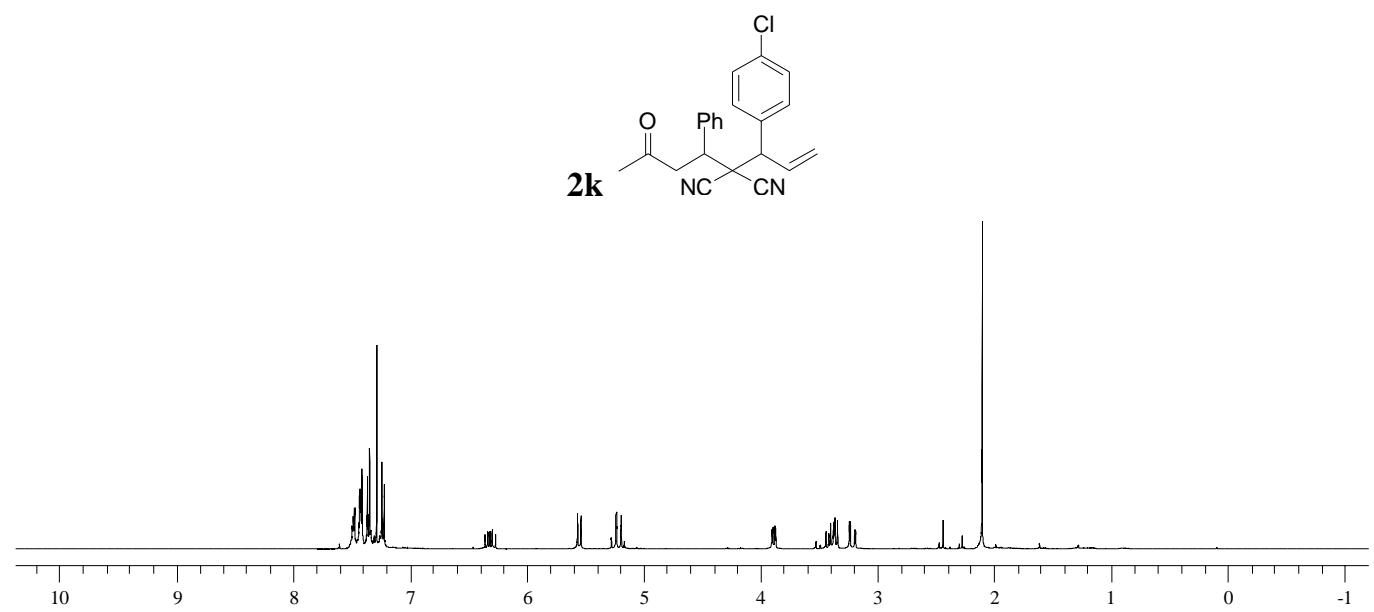



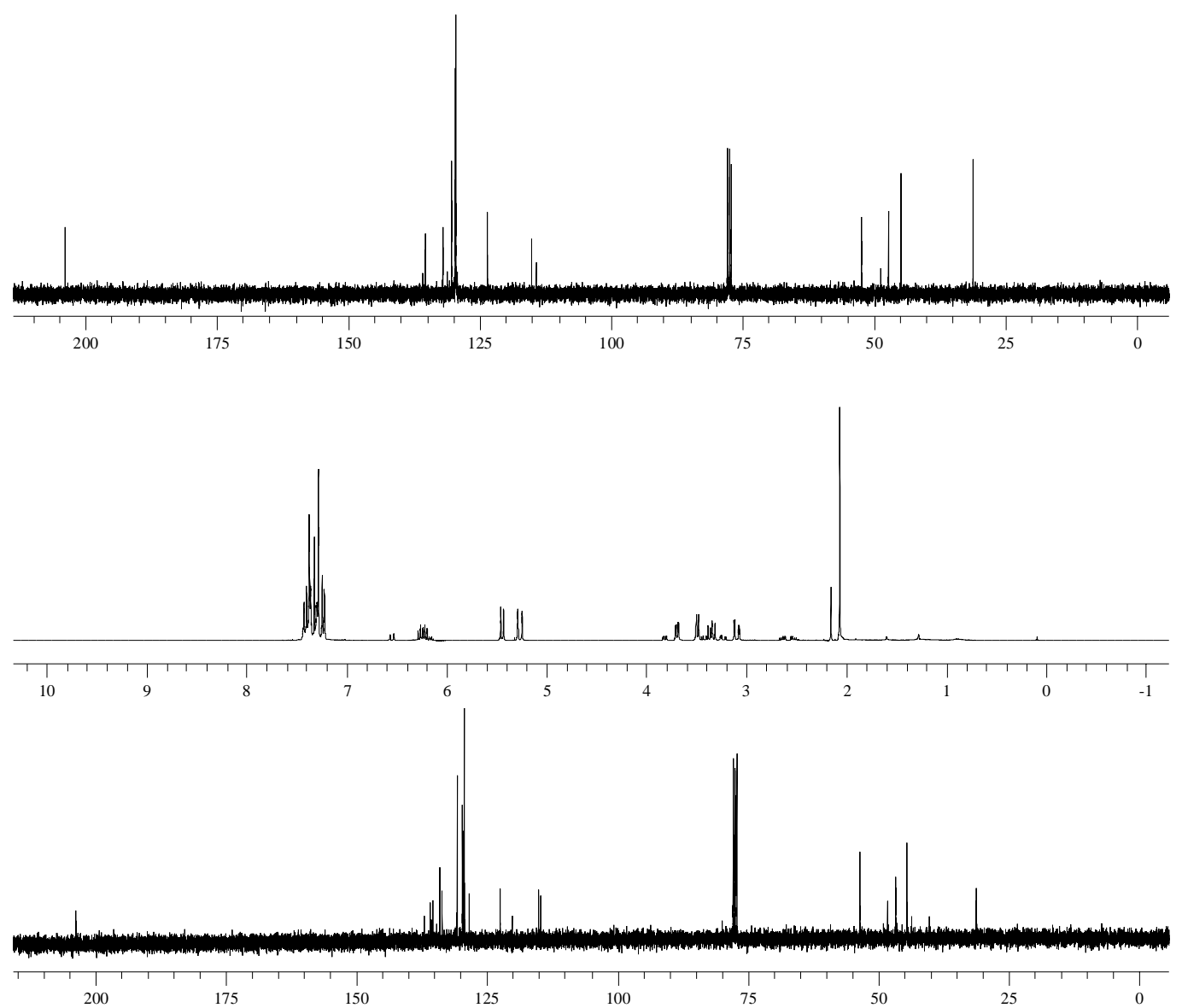

2k: Oil: ${ }^{1} \mathrm{H}$ NMR (400 MHz, $\left.\mathrm{CDCl}_{3}\right)$ Major diastereomer: $\delta$ 7.50-7.22 (m, 9H: arom $\left.\mathrm{H}\right), 6.31(\mathrm{~m}$, $1 \mathrm{H}, J=16.7,10.4,9.1 \mathrm{~Hz}:=\mathrm{CH}), 5.55\left(\mathrm{~d}, 1 \mathrm{H}, J=10.4 \mathrm{~Hz}: \mathrm{CH}=\mathrm{CH}(H)_{\mathrm{E}}\right), 5.21(\mathrm{~d}, 1 \mathrm{H}, J=16.7 \mathrm{~Hz}$ : $\left.\mathrm{CH}=\mathrm{CH}(H)_{\mathrm{Z}}\right), 3.88\left(\mathrm{dd}, J=10.6,3.0 \mathrm{~Hz}, 1 \mathrm{H}: \mathrm{C}(\mathrm{O}) \mathrm{CH}_{2} \mathrm{CHPh}\right), 3.44-3.34(\mathrm{~m}, 2 \mathrm{H}$ : overlapping CHAr, $\left.\mathrm{C}(\mathrm{O}) \mathrm{CH}_{2}\right), 3.21\left(\mathrm{dd}, J=17.4,3.3 \mathrm{~Hz}, 1 \mathrm{H}: \mathrm{C}(\mathrm{O}) \mathrm{CH}_{2}\right), 2.10$ (s, 3H: $\left.\mathrm{C}(\mathrm{O}) \mathrm{CH}_{3}\right) ;{ }^{13} \mathrm{C} \mathrm{NMR}$ $\left(100 \mathrm{MHz}, \mathrm{CDCl}_{3}\right.$ ) Major isomer: $\delta 203.83(\mathbf{C}=\mathrm{O}), 135.86$ (Quat.), 135.42 (Quat.), $131.98(=\mathbf{C H}$ ), 130.30 (Arom. $\mathbf{C H}$ ), 129.54 (Arom. $\mathbf{C H}), 123.56 \quad\left(=\mathrm{CH}_{2}\right), 115.06(\mathbf{C N}), 114.25(\mathbf{C N}), 52.29$ $(\mathbf{C H A r}), 48.65\left(\mathbf{C}(\mathrm{CN})_{2}\right), 47.17\left(\mathrm{C}(\mathrm{O}) \mathbf{C H}_{2}\right), 44.79(\mathbf{C H P h}), 31.12\left(\mathrm{CH}_{3}\right)$. IR $(\mathrm{CH} 2 \mathrm{Cl} 2)$ : vmax 2253,1726,1425, 990, 912. HRMS calcd for C21H20N1O1Cl [M+H-HCN] 337.1, found 337.0.

Minor diastereomer: $\delta$ 7.43-7.22 (m, 9H: arom $\mathrm{H}), 6.24(\mathrm{~m}, 1 \mathrm{H}, J=16.7,10.4,9.1 \mathrm{~Hz}:=\mathrm{CH})$, $5.44\left(\mathrm{~d}, 1 \mathrm{H}, J=10.4 \mathrm{~Hz}: \mathrm{CH}=\mathrm{CH}(H)_{\mathrm{E}}\right), 5.26\left(\mathrm{~d}, 1 \mathrm{H}, J=16.7 \mathrm{~Hz}: \mathrm{CH}=\mathrm{CH}(H)_{\mathrm{z}}\right), 3.69(\mathrm{dd}, J=10.6$, $3.0 \mathrm{~Hz}, 1 \mathrm{H}: \mathrm{C}(\mathrm{O}) \mathrm{CH}_{2} \mathrm{CHPh}$ ), 3.49 (d, $1 \mathrm{H}, J=8.8 \mathrm{~Hz}: \mathrm{CHAr}$ ), 3.35 (dd, $J=17.2,10.4 \mathrm{~Hz}, 1 \mathrm{H}$ : $\left.\mathrm{C}(\mathrm{O}) \mathrm{CH}_{2}\right), 3.10$ (dd, $\left.J=16.9,3.0 \mathrm{~Hz}, 1 \mathrm{H}: \mathrm{C}(\mathrm{O}) \mathrm{CH}_{2}\right), 2.07$ (s, $\left.3 \mathrm{H}: \mathrm{C}(\mathrm{O}) \mathrm{CH}_{3}\right) ;{ }^{13} \mathrm{C} \mathrm{NMR}(100 \mathrm{MHz}$ $\left.\mathrm{CDCl}_{3}\right) \delta 203.78(\mathbf{C}=\mathrm{O}), 135.91$ (Quat.), 135.39 (Quat.), $133.56(=\mathbf{C H}), 130.70-128.22$ (Arom. $\mathbf{C H}), 122.39\left(=\mathrm{CH}_{2}\right), 115.14(\mathbf{C N}), 114.63(\mathbf{C N}), 53.57(\mathbf{C H A r}), 48.21\left(\mathbf{C}(\mathrm{CN})_{2}\right), 46.62$ $\left(\mathrm{C}(\mathrm{O}) \mathrm{CH}_{2}\right), 44.53(\mathbf{C H P h}), 31.19\left(\mathrm{CH}_{3}\right)$. 


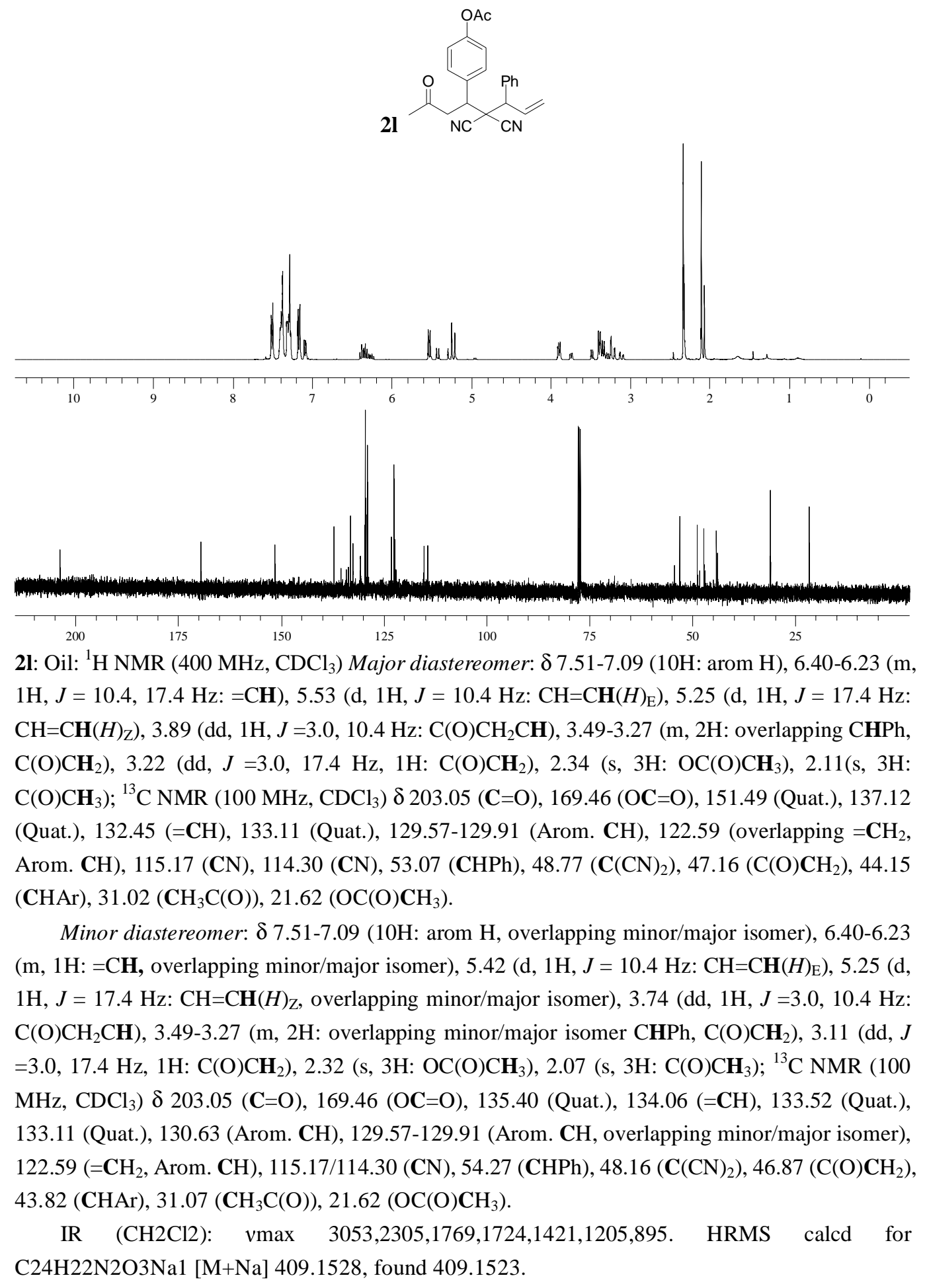




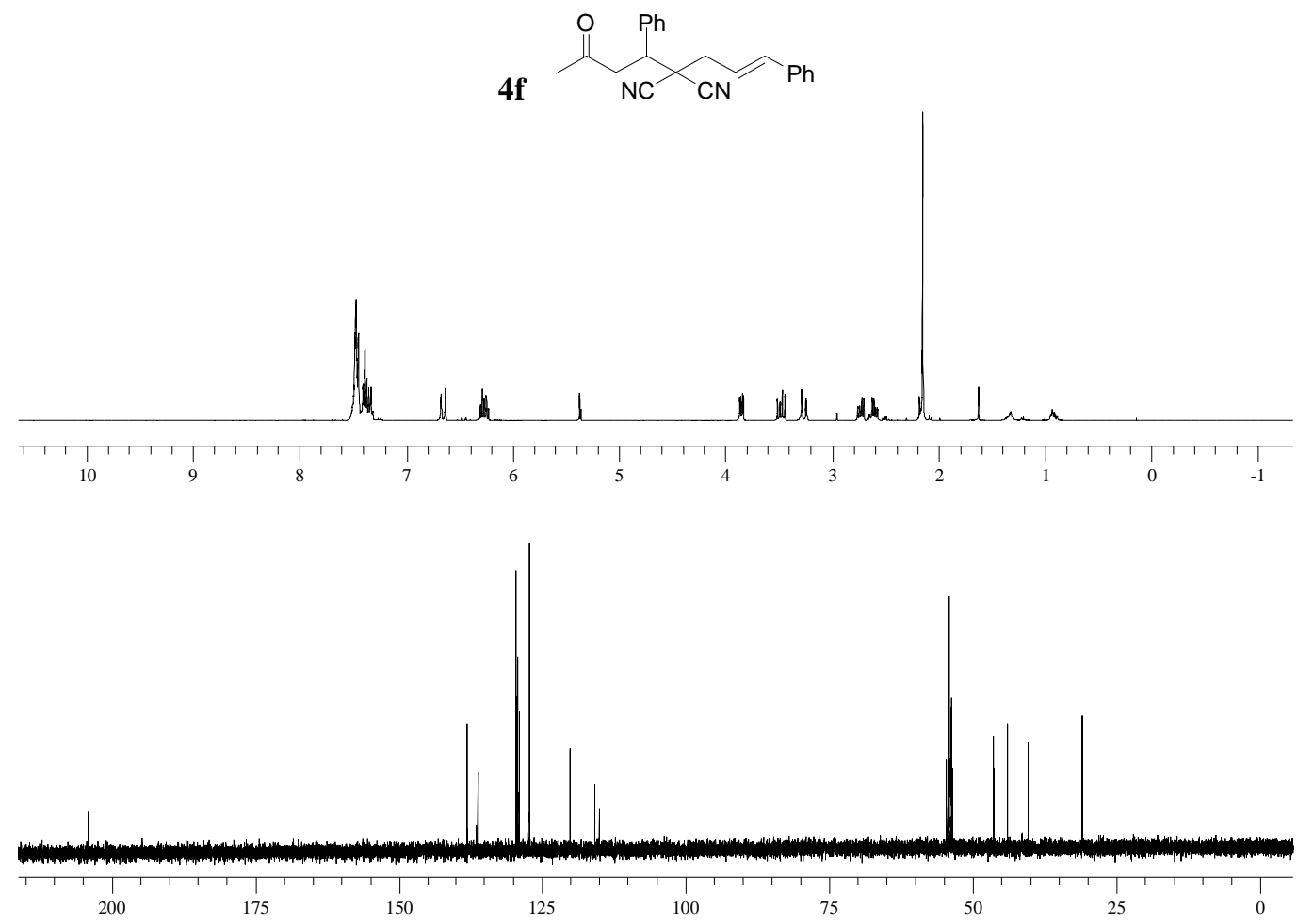

4f: Oil: ${ }^{1} \mathrm{H}$ NMR $\left(400 \mathrm{MHz}, \mathrm{CD}_{2} \mathrm{Cl}_{2}\right) \delta 7.48-7.33(\mathrm{~m}, 10 \mathrm{H}$ : arom $\mathrm{H}), 6.65(\mathrm{~d}, 1 \mathrm{H}, J=15.7 \mathrm{~Hz}$ : $=\mathrm{CHPh}), 6.26\left(\mathrm{~m}, 1 \mathrm{H}, J=15.7,7.6,7.3 \mathrm{~Hz}: \mathrm{CH}_{2} \mathrm{CH}=\right), 3.85(\mathrm{dd}, 1 \mathrm{H}, J=10.4,3.5 \mathrm{~Hz}$ : $\mathrm{C}(\mathrm{O}) \mathrm{CH}_{2} \mathrm{CHPh}$ ), 3.48 (dd, $\left.J=17.4,10.4 \mathrm{~Hz}, 1 \mathrm{H}: \mathrm{C}(\mathrm{O}) \mathrm{CH}_{2}\right), 3.26(\mathrm{dd}, J=17.4,3.5 \mathrm{~Hz}, 1 \mathrm{H}$ : $\left.\mathrm{C}(\mathrm{O}) \mathrm{CH}_{2}\right), 2.73\left(\mathrm{dd}, J=7.6,13.9 \mathrm{~Hz}, 1 \mathrm{H}: \mathrm{CH}_{2} \mathrm{CH}=\right), 2.60\left(\mathrm{dd}, J=7.3,13.9 \mathrm{~Hz}, 1 \mathrm{H}: \mathrm{CH}_{2} \mathrm{CH}=\right)$, 2.15 (s, 3H: C(O)CH $\left.\mathbf{C H}_{3}\right) ;{ }^{13} \mathrm{C} \mathrm{NMR}\left(100 \mathrm{MHz}, \mathrm{CD}_{2} \mathrm{Cl}_{2}\right) \delta 203.96(\mathbf{C}=\mathrm{O}), 137.99$ (=CHPh), 136.19 (Quat.), 136.19 (Quat.), 129.57 (Arom. CH), 129.50 (Arom. CH), 129.46 (Arom. CH), 129.17 (Arom. CH), 128.90 (Arom. CH), 127.14 (Arom. CH), $122.07\left(\mathrm{CH}_{2} \mathbf{C H}=\right), 115.78(\mathbf{C N}), 115.00$ $(\mathbf{C N}), 46.35(\mathbf{C H P h}), 46.20\left(\mathrm{C}(\mathrm{O}) \mathbf{C H}_{2} \mathrm{CHPh}\right), 43.88\left(\mathbf{C}(\mathrm{CN})_{2}\right), 40.26\left(\mathbf{C H}_{2} \mathrm{CH}=\right), 30.87\left(\mathrm{CH}_{3}\right) . \mathrm{IR}$ (CH2Cl2): vmax 2248,1724,1456,968. HRMS calcd for C44H40N4O2Na1 [2M+Na] 679.3049, found 679.3074 .

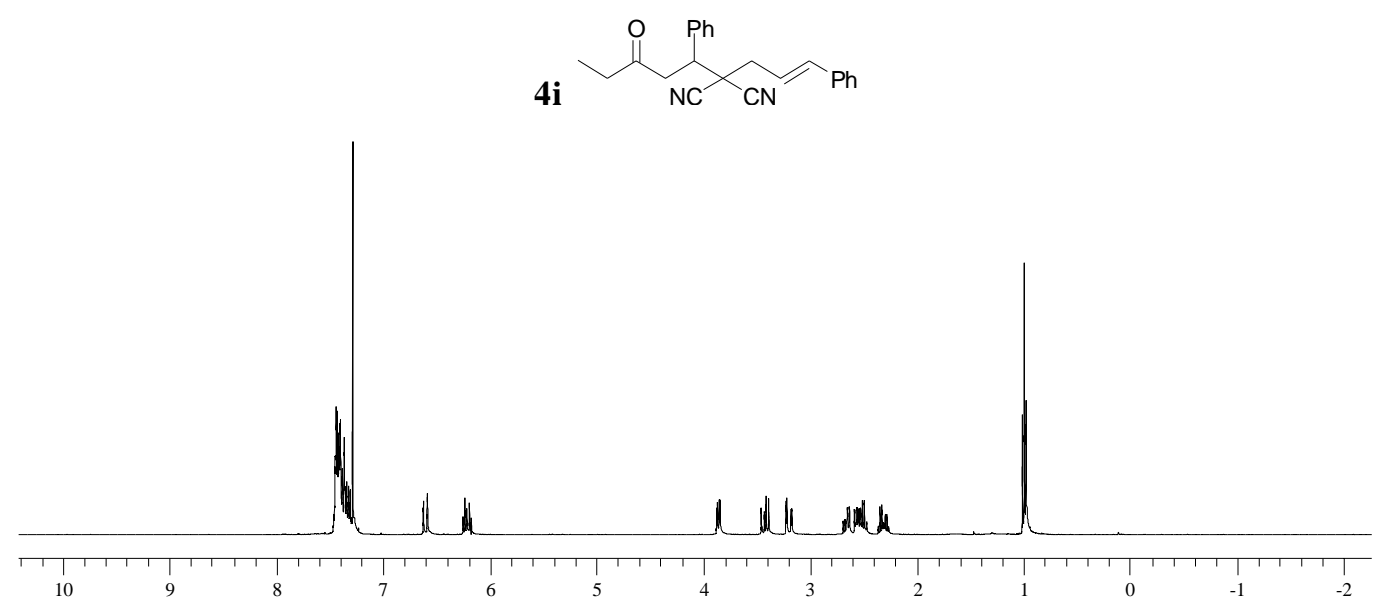




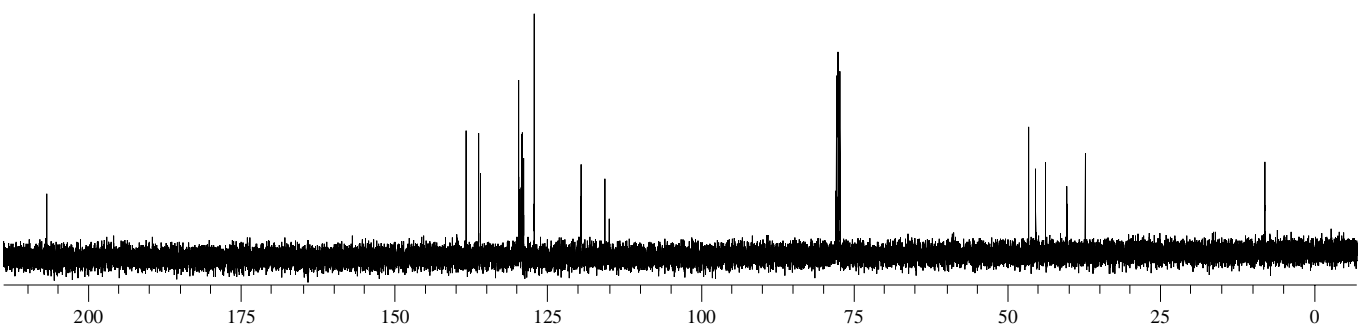

4i: Oil: ${ }^{1} \mathrm{H}$ NMR $\left(400 \mathrm{MHz}, \mathrm{CDCl}_{3}\right) \delta$ 7.44-7.31 (m, $10 \mathrm{H}$ : arom H), $6.60(\mathrm{~d}, 1 \mathrm{H}, J=15.7 \mathrm{~Hz}$ : $=\mathrm{CHPh}), 6.21(\mathrm{~m}, 1 \mathrm{H}, J=15.7,7.8,7.3 \mathrm{~Hz}: \mathbf{C H}=\mathrm{CHPh}), 3.86(\mathrm{dd}, 1 \mathrm{H}, J=10.1,3.5 \mathrm{~Hz}:$ $\mathrm{C}(\mathrm{O}) \mathrm{CH}_{2} \mathrm{CHPh}$ ), 3.42 (dd, $1 \mathrm{H}, J=10.1,17.1 \mathrm{~Hz}: \mathrm{EtC}(\mathrm{O}) \mathrm{CH}_{2}$ ), 3.20 (dd, $1 \mathrm{H}, J=3.5,17.1 \mathrm{~Hz}$ : $\left.\mathrm{EtC}(\mathrm{O}) \mathrm{CH}_{2}\right), 2.66$ (dd, $\left.J=13.9,7.8 \mathrm{~Hz}, 1 \mathrm{H}: \mathrm{CH}_{2} \mathrm{CH}=\right)$, 2.58-2.47 (m, 2H: overlapping $\mathrm{CH}_{2} \mathrm{CH}=$, $\mathrm{CH}_{2} \mathrm{C}(\mathrm{O})$ ), 2.31 (q, $\left.1 \mathrm{H}, J=7.3 \mathrm{~Hz}: \mathrm{CH}_{2} \mathrm{C}(\mathrm{O})\right), 0.95$ (t, 3H, $\left.J=7.3 \mathrm{~Hz}: \mathrm{C}(\mathrm{O}) \mathrm{CH}_{2} \mathrm{CH}_{3}\right) ;{ }^{13} \mathrm{C} \mathrm{NMR}$ $\left(100 \mathrm{MHz}, \mathrm{CDCl}_{3}\right) \delta 206.77(\mathbf{C}=\mathrm{O}), 138.27 \quad(=\mathbf{C H P h}), 136.15$ (Quat.), 135.82 (Quat.), 129.64-128.87 (Arom. CH), 127.12 (Arom. $\mathbf{C H}), 119.53(\mathbf{C H}=\mathrm{CHPh}), 115.67(\mathbf{C N}), 114.90(\mathbf{C N})$, $46.47(\mathbf{C H P h}), 45.30\left(\mathrm{EtC}(\mathrm{O}) \mathbf{C H}_{2}\right) 43.68\left(\mathbf{C}(\mathrm{CN})_{2}\right), 40.22\left(\mathbf{C H}_{2} \mathbf{C H}=\right), 37.20\left(\mathbf{C H}_{2} \mathrm{C}(\mathrm{O})\right), 7.87$ $\left(\mathrm{CH}_{3}\right)$. IR (CH2Cl2): vmax 2249,1720,1456,968. HRMS calcd for C23H23N2O1 [M+H] 343.1810, found 343.1830 .

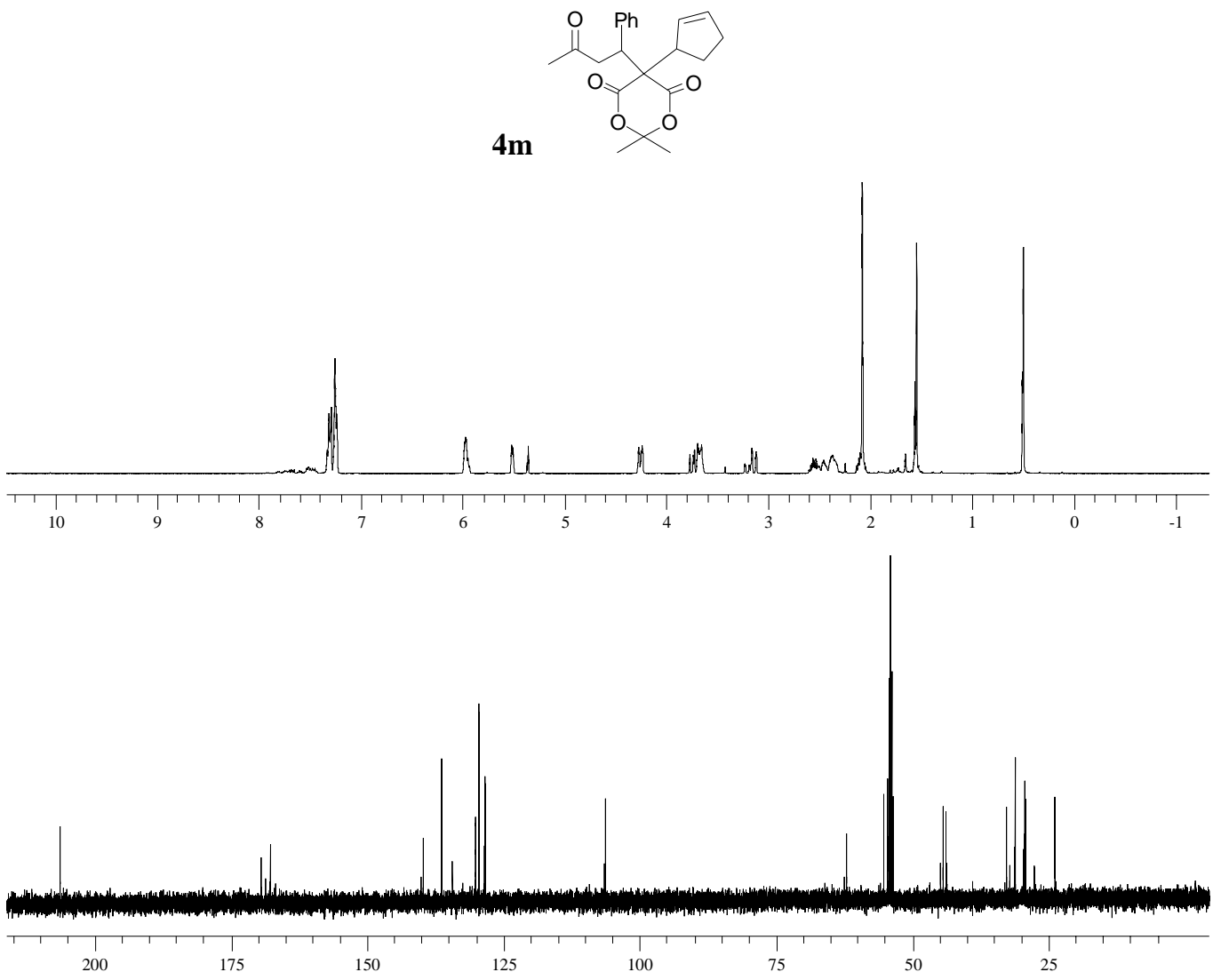

4m: Oil: ${ }^{1} \mathrm{H}$ NMR (400 MHz, $\left.\mathrm{CDCl}_{3}\right)$ Major diastereomer: $\delta 7.31(\mathrm{~m}, 2 \mathrm{H}$ : arom $\mathrm{H}), 7.25(\mathrm{~m}, 2 \mathrm{H}$ : $\operatorname{arom~} \mathrm{H}), 7.24(\mathrm{~m}, 1 \mathrm{H}$ : arom $\mathrm{H}), 5.97(\mathrm{~m}, 1 \mathrm{H},=\mathrm{CH}), 5.51(\mathrm{~m}, 1 \mathrm{H},=\mathrm{CH}), 4.26(\mathrm{dd}, 1 \mathrm{H}, J=11.6,3.0$ Hz: $\mathrm{CHPh}), 3.77-3.63$ (m, 2H: overlapping $\left.\mathrm{CHCH}=\mathrm{CH}, \mathrm{C}(\mathrm{O}) \mathrm{CH}_{2}\right), 3.14(\mathrm{dd}, J=2.0,17.4 \mathrm{~Hz}, 1 \mathrm{H}$ : 
$\left.\mathrm{C}(\mathrm{O}) \mathrm{CH}_{2}\right), 2.56$ (m, 1H: $\left.\mathbf{C H}_{2} \mathrm{CHCH}=\mathrm{CH}\right), 2.46$ (m, 1H: $\left.\mathrm{CH}_{2} \mathrm{CH}=\right), 2.37$ (m, 1H: $\left.\mathrm{CH}_{2} \mathrm{CH}=\right), 2.08$ (m, 1H: $\mathbf{C H}_{2} \mathrm{CHCH}=\mathrm{CH}$ ), 2.08 (s, 3H: $\mathrm{C}(\mathrm{O}) \mathrm{CH}_{3}$ ), 1.54 (s, 3H: $\mathrm{CH}_{3}$ ), 0.50 (s, 3H: $\mathrm{CH}_{3}$ ); ${ }^{13} \mathrm{C}$ NMR (100 MHz, $\left.\mathrm{CDCl}_{3}\right) \delta 206.31$ (C=O), 169.40 (OC=O) 167.72 (OC=O), 139.78 (Quat.), 136.36 (=CH: $\delta=5.97 \mathrm{ppm}$ ), 130.14 (Arom. $\mathbf{C H}$ ), 129.46 (Arom. $\mathbf{C H}), 128.37(=\mathbf{C H}), 106.27$ $\left(\mathbf{C}\left(\mathrm{CH}_{3}\right)_{2}\right), 62.11\left(\mathbf{C}\left(\mathrm{CO}_{2} \mathrm{R}\right)_{2}\right), 55.19(\mathbf{C H C H}=\mathrm{CH}), 44.27(\mathbf{C H P h}), 43.87\left(\mathrm{C}(\mathrm{O}) \mathbf{C H}_{2}\right), 32.73$ $\left(\mathbf{C H}_{2} \mathrm{CH}=\right), 29.61\left(\mathrm{CH}_{3}\right), 29.37\left(\mathrm{CH}_{3}\right), 29.16\left(\mathbf{C H}_{3}\right), 23.85\left(\mathbf{C H}_{2} \mathrm{CHCH}=\mathrm{CH}\right)$.

Minor diastereomer: $\delta$ 7.31-7.24 $(\mathrm{m}, 5 \mathrm{H}$ : arom $\mathrm{H}), 5.97(\mathrm{~m}, 1 \mathrm{H}:=\mathrm{CH}$, overlapping minor/major isomer), $5.36(\mathrm{~m}, 1 \mathrm{H},=\mathrm{CH}), 4.26(\mathrm{dd}, 1 \mathrm{H}: \mathrm{CHPh}$, overlapping minor/major isomer), 3.77-3.63 (m, 2H: overlapping minor/major isomer $\left.\mathbf{C H C H}=\mathrm{CH}, \mathrm{C}(\mathrm{O}) \mathrm{CH}_{2}\right), 3.14\left(1 \mathrm{H}: \mathrm{C}(\mathrm{O}) \mathrm{CH}_{2}\right.$, overlapping minor/major isomer), $2.56\left(1 \mathrm{H}: \mathrm{CH}_{2} \mathrm{CHCH}=\mathrm{CH}\right.$, overlapping minor/major isomer), $2.46\left(1 \mathrm{H}: \mathbf{C H}_{2} \mathrm{CH}=\right.$, overlapping minor/major isomer), $2.37\left(1 \mathrm{H}: \mathrm{CH}_{2} \mathrm{CH}=\right.$, overlapping minor/major isomer), 2.08 (1H: $\mathrm{CH}_{2} \mathrm{CHCH}=\mathrm{CH}$, overlapping minor/major isomer), 2.08 (s, $3 \mathrm{H}$ : $\mathrm{C}(\mathrm{O}) \mathrm{CH}_{3}$, overlapping minor/major isomer), 1.54 (s, $3 \mathrm{H}: \mathrm{CH}_{3}$, overlapping minor/major isomer), 0.50 (s, 3H: $\mathrm{CH}_{3}$, overlapping minor/major isomer); ${ }^{13} \mathrm{C} \mathrm{NMR}\left(100 \mathrm{MHz}, \mathrm{CDCl}_{3}\right) \delta 206.31$ $(\mathbf{C}=\mathrm{O}), 168.65(\mathrm{OC}=\mathrm{O}), 166.77(\mathrm{OC}=\mathrm{O}), 140.70$ (Quat.), $134.36(=\mathrm{CH}), 130.14,129.46$ (Arom. $\left.\mathbf{C H}), 128.37(=\mathbf{C H}), 106.51\left(\mathbf{C}\left(\mathrm{CH}_{3}\right)_{2}\right), 62.48\left(\mathrm{C}_{\left(\mathrm{CO}_{2} \mathrm{R}\right.}\right)_{2}\right), 55.19(\mathbf{C H C H}=\mathrm{CH}), 44.78(\mathbf{C H P h})$, $43.69\left(\mathrm{C}(\mathrm{O}) \mathbf{C H}_{2}\right), 32.09\left(\mathbf{C H}_{2} \mathrm{CH}=\right), 29.61,29.37,29.16\left(\mathrm{CH}_{3}\right), 27.63\left(\mathrm{CH}_{2} \mathbf{C H C H}=\mathrm{CH}\right)$.

IR (CH2Cl2): vmax 3063, 1765,1732,1456, 945. HRMS calcd for C21H24N2O5Na1 [M+Na] 379.1521 , found 379.1511 .

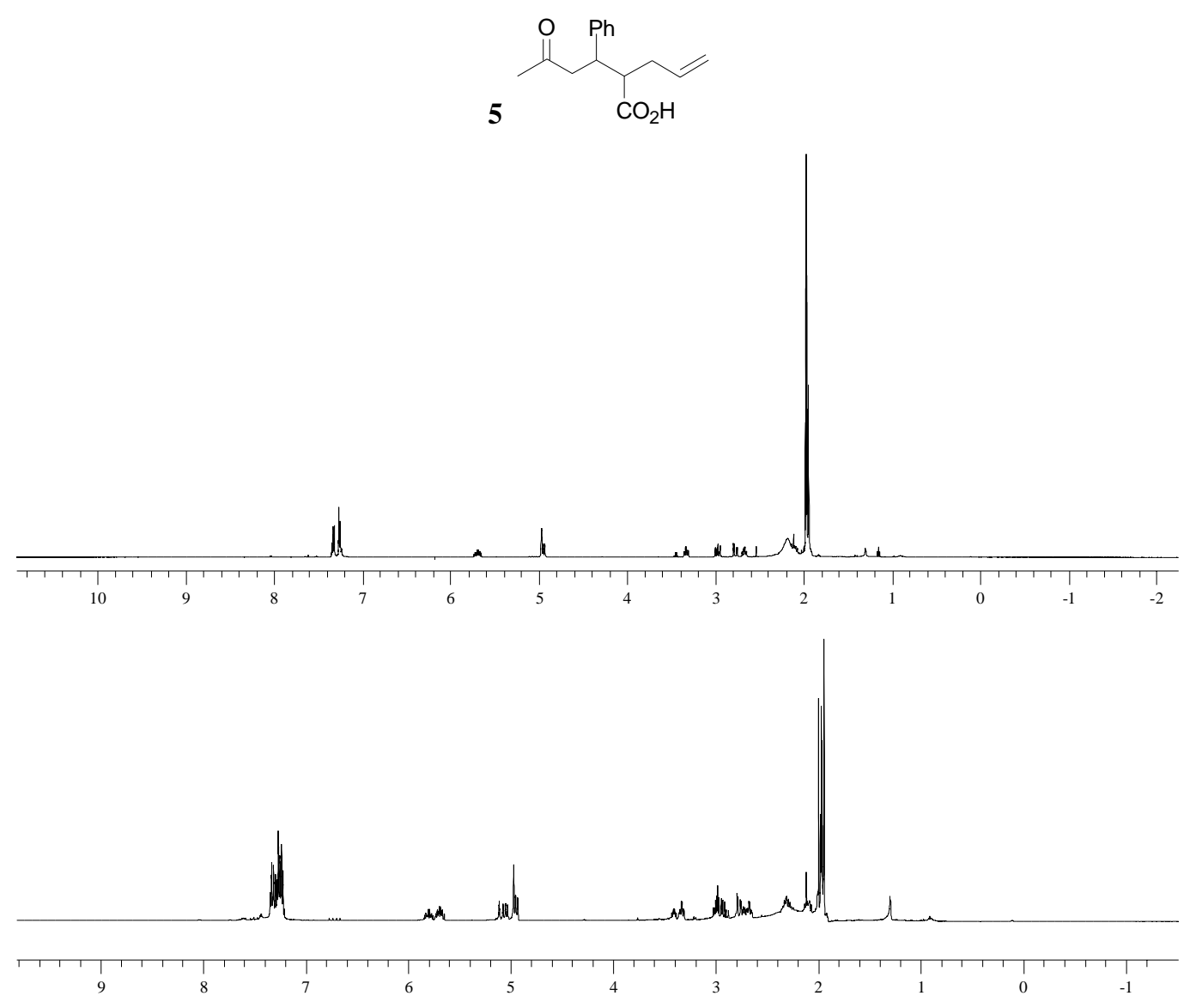




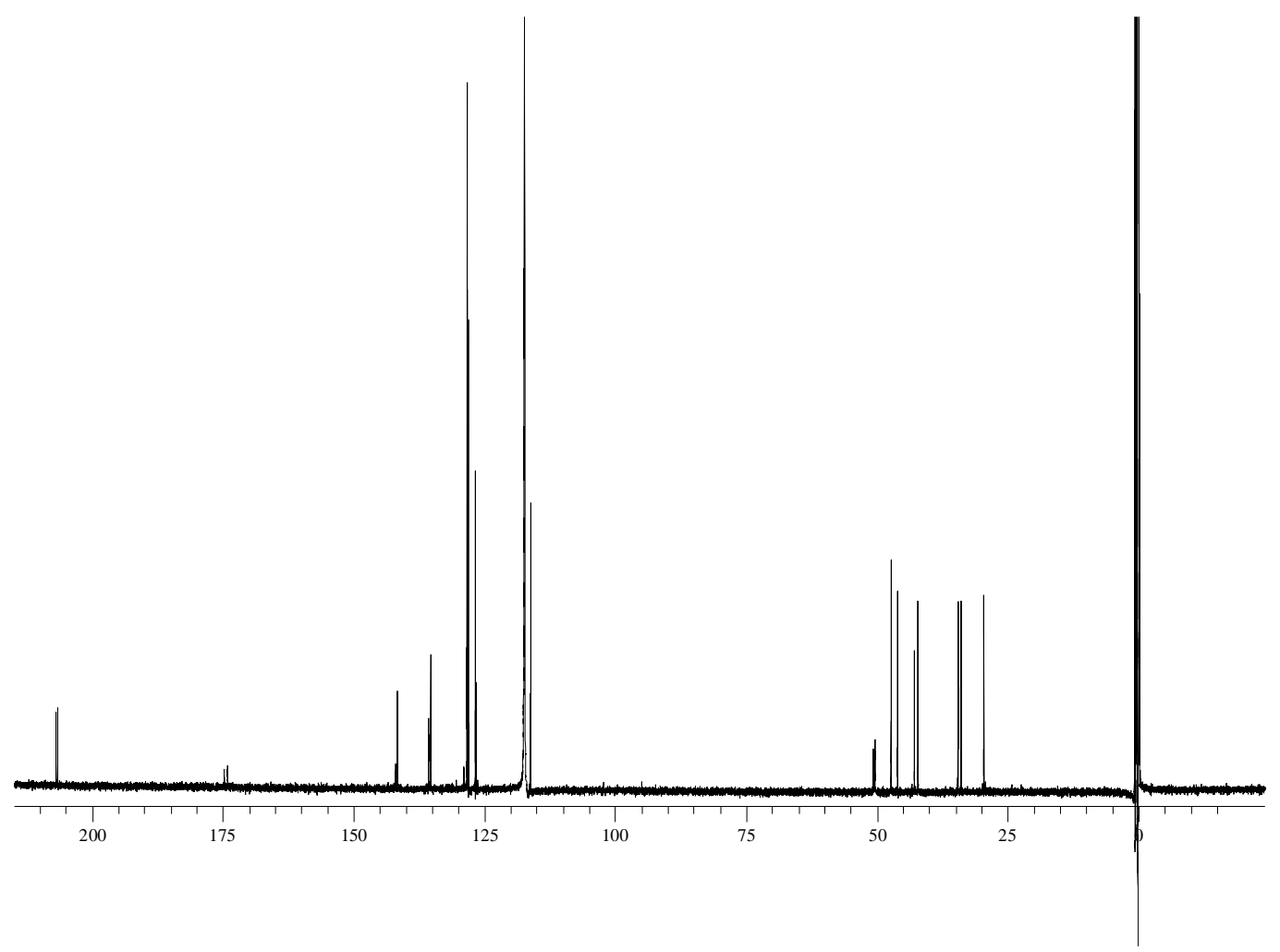

5: Oil: ${ }^{1} \mathrm{H}$ NMR $\left(500 \mathrm{MHz}, \mathrm{CD}_{3} \mathrm{CN}\right)$ Major diastereomer: $\delta 7.33(\mathrm{~m}, 2 \mathrm{H}$ : arom $\mathrm{H}), \delta 7.26(\mathrm{~m}, 3 \mathrm{H}$ : arom $\mathrm{H}), \delta 5.70(\mathrm{~m}, 1 \mathrm{H}, J=7.3 \mathrm{~Hz}:=\mathrm{CH}), 4.97\left(\operatorname{app~s}, 1 \mathrm{H}, \mathrm{CH}=\mathrm{CH}(H)_{\mathrm{Z}}\right), 4.94(\mathrm{~d}, 1 \mathrm{H}, J=7.3 \mathrm{~Hz}$ : $\left.\mathrm{CH}=\mathrm{CH}(H)_{\mathrm{E}}\right), 3.33\left(\mathrm{td}, 1 \mathrm{H}, J=10.4,3.8 \mathrm{~Hz}: \mathrm{C}(\mathrm{O}) \mathrm{CH}_{2} \mathrm{CHPh}\right), 2.98(\mathrm{dd}, 1 \mathrm{H}, J=10.4,16.4 \mathrm{~Hz}$ : $\left.\mathrm{C}(\mathrm{O}) \mathrm{CH}_{2}\right), 2.77\left(\mathrm{dd}, 1 \mathrm{H}, J=3.8,16.4 \mathrm{~Hz}: \mathrm{C}(\mathrm{O}) \mathrm{CH}_{2}\right), 2.67\left(\mathrm{td}, J=4.1,1 \mathrm{H}, 10.1 \mathrm{~Hz}: \mathrm{CHCO}_{2} \mathrm{H}\right)$, 2.10 (m, 1H: overlapping $\left.\mathrm{H}_{2} \mathrm{O}, \mathrm{CH}_{2} \mathrm{CH}=\right), 1.97$ (3H: overlapping $\left.\mathrm{CD}_{3} \mathrm{CN}, \mathrm{CH}_{2} \mathrm{CH}=\right), 1.97(3 \mathrm{H}$ : overlapping $\left.\mathrm{CD}_{3} \mathrm{CN}, \mathrm{C}(\mathrm{O}) \mathrm{CH}_{3}\right) ;{ }^{13} \mathrm{C}$ NMR $\left(125 \mathrm{MHz}, \mathrm{CD}_{3} \mathrm{CN}\right) \delta 206.26(\mathbf{C}=\mathrm{O}), 174.34(\mathrm{HOC}=\mathrm{O})$, 141.41 (Quat), 135.03 (=CH), 128.07 (Arom. $\mathbf{C H}$ ), 126.47 (Arom. $\mathbf{C H}$ ), $115.82\left(=\mathbf{C H}_{2}\right), 50.31$ $\left(\mathbf{C H C O}_{2} \mathrm{H}\right), 46.96\left(\mathrm{C}(\mathrm{O}) \mathbf{C H}_{2}\right), 42.52(\mathbf{C H P h}), 34.20\left(\mathbf{C H}_{2} \mathbf{C H}=\right), 29.26\left(\mathbf{C H}_{3}\right)$.

Minor diastereomer: $\delta 7.35-7.23(\mathrm{~m}, 5 \mathrm{H}$ : arom $\mathrm{H}$, overlapping minor/major isomer), $\delta 5.80(\mathrm{~m}$, $1 \mathrm{H}, J=17.2,9.9 \mathrm{~Hz}:=\mathrm{CH}), 5.06\left(\mathrm{~d}, 1 \mathrm{H}, J=17.2 \mathrm{~Hz}, \mathrm{CH}=\mathrm{CH}(H)_{\mathrm{Z}}\right), 5.04(\mathrm{~d}, 1 \mathrm{H}, J=9.9 \mathrm{~Hz}$ : $\left.\mathrm{CH}=\mathrm{CH}(H)_{\mathrm{E}}\right), 3.42\left(\mathrm{td}, 1 \mathrm{H}, \quad J=9.2,4.8 \mathrm{~Hz}: \mathrm{C}(\mathrm{O}) \mathrm{CH}_{2} \mathrm{CHPh}\right), 3.03-2.88\left(\mathrm{~m}, 1 \mathrm{H}: \mathrm{C}(\mathrm{O}) \mathrm{CH}_{2}\right.$, overlapping minor/major isomer), 2.80-2.65 (m, $2 \mathrm{H}$ : overlapping minor/major isomer $\mathrm{C}(\mathrm{O}) \mathrm{CH}_{2}$, $\mathrm{CHCO}_{2} \mathrm{H}$ ), 2.32 (m, 2H: overlapping $\mathrm{H}_{2} \mathrm{O}, \mathrm{CH}_{2} \mathrm{CH}=$ ), 1.97 (3H: overlapping minor/major isomer $\left.\mathrm{CD}_{3} \mathrm{CN}, \mathrm{C}(\mathrm{O}) \mathrm{CH}_{3}\right) ;{ }^{13} \mathrm{C}$ NMR $\left(125 \mathrm{MHz}, \mathrm{CD}_{3} \mathrm{CN}\right) \delta 206.85(\mathbf{C}=\mathrm{O}), 174.97(\mathrm{HOC}=\mathrm{O}), 142.01$ (Quat.), $135.59(=\mathrm{CH}), 128.22$ (Arom. $\mathbf{C H}$ ), 126.62 (Arom. CH), $116.24\left(=\mathrm{CH}_{2}\right), 50.29$ $\left(\mathbf{C H C O}_{2} \mathrm{H}\right), 45.98\left(\mathrm{C}(\mathrm{O}) \mathbf{C H}_{2}\right), 42.12(\mathbf{C H P h}), 33.85\left(\mathbf{C H}_{2} \mathbf{C H}=\right), 29.53\left(\mathbf{C H}_{3}\right) . \quad$ IR $(\mathrm{CH} 2 \mathrm{Cl} 2)$ : vmax 3413, 1745,1711. HRMS calcd for C15H18O3Na1 [M+Na] 269.1154, found 269.1154.

\footnotetext{
${ }^{1}$ Shim, J. G.; Nakamura, H.; Yamamoto, Y, J. Org. Chem. 1998, 63, 8470-8474.
} 